\title{
CARLEMAN ESTIMATE FOR ELLIPTIC OPERATORS WITH COEFFICIENTS WITH JUMPS AT AN INTERFACE IN ARBITRARY DIMENSION AND APPLICATION TO THE NULL CONTROLLABILITY OF LINEAR PARABOLIC EQUATIONS
}

\author{
JÉRÔME LE ROUSSEAU AND LUC ROBBIANO
}

\begin{abstract}
In a bounded domain of $\mathbf{R}^{n+1}, n \geq 2$, we consider a second-order elliptic operator, $A=-\partial_{x_{0}}^{2}-\nabla_{x}$. $\left(c(x) \nabla_{x}\right.$ ), where the (scalar) coefficient $c(x)$ is piecewise smooth yet discontinuous across a smooth interface $S$. We prove a local Carleman estimate for $A$ in the neighborhood of any point of the interface. The "observation" region can be chosen independently of the sign of the jump of the coefficient $c$ at the considered point. The derivation of this estimate relies on the separation of the problem into three microlocal regions and the Calderón projector technique. Following the method of Lebeau and Robbiano [LR95] we then prove the null controllability for the linear parabolic initial problem with Dirichlet boundary conditions associated to the operator $\partial_{t}-\nabla_{x} \cdot\left(c(x) \nabla_{x}\right)$.
\end{abstract}

Keywords: Elliptic equation; Non-smooth coefficient; Transmission problem; Carleman estimate; Microlocal analysis; Calderón projectors; Parabolic equation; Control.

AMS 2000 subject classification: 35J15; 35S15; 35K05; 93B05; 93B07.

\section{INTRODUCTION AND NOTATION}

The question of the null controllability of linear parabolic partial differential equations with smooth coefficients was solved in the 1990's [LR95, FI96]. In the case of discontinuous coefficients in the principal part of the parabolic operator, the controllability issue and its dual counterpart, observability, are not fully solved yet. A result of controllability for a semi-linear heat equation with a coefficient that is discontinuous at an interface was proven in [DOP02] by means of a global Carleman observability estimate. Roughly speaking, as in the case of hyperbolic systems (see e.g. [Lio88, page 356]), the authors of [DOP02] proved their controllability result in the case where the control is supported in the region where the diffusion coefficient is the 'lowest'. In both cases, however, the approximate controllability, and its dual counterpart, uniqueness, are true without any restriction on the monotonicity of the coefficients. It is then natural to question whether or not an observability estimate holds in the case of non-smooth coefficients and arbitrary observation location.

Recently, in the one-dimensional case, the controllability result for parabolic equations was proven for general piecewise $\mathscr{C}^{1}$ coefficients in [BDL07a], and for coefficients with bounded variations $(B V)$ in [Le 07], which improved the result of [FCZ02]. The proof relies on global Carleman estimates, which moreover allow to treat semilinear equations. Simultaneously, a controllability result for parabolic equations with

Date: March 27, 2009. 
general bounded coefficients in one dimension was proven in [AE07]. The method used there to achieve null controllability is that of [LR95], which limits the field of applications to linear equations.

In the $n$-dimensional case, $n \geq 2$, a positive answer to the controllability question was given for a class of discontinuous coefficients, with separated variables, that are smooth w.r.t. to all but one variables, which includes the case of stratified media [BDL07b]. The proof relies both on the Carleman estimates of [BDL07a, Le 07] in the one-dimensional case and the method of [LR95].

In the present article, in the case $n \geq 2$, we achieve null controllability for a linear parabolic equation in the case of a coefficient that exhibits jumps of arbitrary signs at an interface. Let $\Omega$ be a smooth bounded connected domain in $\mathbf{R}^{n}$. We consider the operator $L:=\nabla_{x} \cdot\left(c(x) \nabla_{x}\right)$, with possibly additional lower-order terms, and where $c(x)$ satisfies

$$
0<c_{\min } \leq c(x) \leq c_{\max }<\infty,
$$

to ensure uniform ellipticity for $L$. The coefficient $c$ is assumed smooth apart from across an interface $S$, where it may jump. The interface $S$ is the boundary of a smooth open subset $\Omega_{1} \Subset \Omega$, i.e., $\Omega_{1}$ lies on one side of $S$. Let $T>0$ and set $Q_{T}=(0, T) \times \Omega$. We set $\Omega_{2}=\Omega \backslash \overline{\Omega_{1}}$. We prove the following null controllability result.

Theorem 1.1. For an arbitrary time $T>0$ and an arbitrary non-empty open subset $\omega \subset \Omega$ and an initial condition $q_{0} \in L^{2}(\Omega)$, there exists $u \in L^{2}((0, T) \times \Omega)$ such that the solution $q$ of

$$
\begin{cases}\partial_{t} q-L q=1_{\omega} u & \text { in } Q_{T}, \\ q(t, x)=0 & \text { on }(0, T) \times \partial \Omega, \\ q(0, x)=q_{0}(x) & \text { in } \Omega,\end{cases}
$$

satisfies $q(T)=0$ a.e. in $\Omega$.

We follow the method of [LR95], thus proving local Carleman estimates for an elliptic operator associated to the considered parabolic problem: we introduce the elliptic operator $A:=-\partial_{x_{0}}^{2}-L$. The variable $x_{0}$ is an additional variables in $\left(0, X_{0}\right)$, for some $X_{0}>0$. We provide such a local Carleman estimate for the operator $A$ in a small neighborhood $V$ of a point $\left(y_{0}, y\right)$ of $\left(0, X_{0}\right) \times S$ with an "observation" on one side of $S$, independently of the sign of the jump of $c$ at $\left(y_{0}, y\right)$. We hence treat all possible cases including the case that can be treated more classically as mentioned above, for which the "observation" is supported in the region where the diffusion coefficient is the 'lowest' [DOP02].

We denote by (., .) the inner product on $L^{2}\left(\left(0, X_{0}\right) \times \Omega\right)$ and by $\|.\|_{0}$ the induced norm. In the present article, we shall make use of techniques from the semi-classical analysis of pseudodifferential operators ( $\psi \mathrm{DOs})$ [Mar02]. With $h$ as the small parameter, we set $D=\frac{h}{i} \partial$. Accordingly, we shall use the semi-classical Sobolev norm $\|f\|_{k}^{2}:=\sum_{|\alpha| \leq k}\left\|D_{x_{0}, x}^{\alpha} f\right\|_{0}^{2}, k \in \mathbf{N}$.

The Carleman estimate we aim to prove is of the form

$$
h\left\|e^{\varphi / h} w\right\|_{0}^{2}+h^{3}\left\|e^{\varphi / h} \nabla_{x_{0}, x} w\right\|_{0}^{2} \leq C h^{4}\left\|e^{\varphi / h} f\right\|_{0}^{2}, \quad A w=f \text { in }\left(0, X_{0}\right) \times(\Omega \backslash S), h>0,
$$

for $h$ sufficiently small and $\operatorname{supp}(w) \subset V$, when $w$ is smooth on both sides of the interface. 
The sign of $\partial_{n} \varphi$ at the interface locates the side of the interface on which the "observation" takes place (see Section 2 for the application of the local Carleman estimate). To achieve such a Carleman estimate we follow the method of [LR97], in the spirit of the work of [Bel03]. In particular, we separate the interface problem into three microlocal regions for which partial Carleman estimates are obtained. In some of these regions we make use of the Calderón-projector technique.

With this local Carleman estimate at the interface, we can then prove an interpolation inequality that first yields an estimation of the loss of orthogonality for the eigenfunctions $\phi_{j}(x), j \in \mathbf{N}$, of the operator $L$, with Dirichlet boundary conditions, when these eigenfunctions are restricted to $\omega$. We denote by $\mu_{j}, j \in \mathbf{N}$, the associated eigenvalues, sorted in an increasing sequence.

Theorem 1.2. For any $\left(a_{j}\right)_{j \in \mathbf{N}} \subset \mathbf{C}$ we have:

$$
\sum_{\mu_{j} \leq \mu}\left|a_{j}\right|^{2} \leq C e^{C \sqrt{\mu}} \int_{\omega}\left|\sum_{\mu_{j} \leq \mu} a_{j} \phi_{j}(x)\right|^{2} d x, \quad \mu>0 .
$$

Following [LR95], this estimation then yields a construction of the control function $u(t, x)$ in (1.1), by sequentially acting on a finite yet increasing number of eigenspaces, and we hence obtain the result of Theorem 1.1. We refer the reader to [LR95] or [LZ98, Section 5, Proposition 2] for the details.

The reader will observe that the proof of the Carleman estimate can be adapted to other elliptic operators with non-smooth coefficients across an interface. Beyond the controllability result of interest in this article, such Carleman estimates have a wide range of applications, including unique continuation properties ([Hör63, Zui83, Hör85a]. See Remark 2.8 for further details.

The result of this article opens perspectives for future research towards the null controllability of semilinear parabolic equations with non smooth coefficient in space dimension $n \geq 2$ and towards more complicated geometrical situations, for instance in the case of coefficients with singularities that do not lie on a smooth interface.

In this article, when the constant $C$ is used, it refers to a constant that is independent of the semi-classical parameter $h$. Its value may however change from one line to another. If we want to keep track of the value of a constant we shall use another letter. We shall use of the notation $\langle\eta\rangle:=\left(1+|\eta|^{2}\right)^{\frac{1}{2}}$. Let us now introduce semi-classical $\psi$ DOs. We denote by $S^{m}\left(\mathbf{R}^{n+1} \times \mathbf{R}^{n+1}\right), S^{m}$ for short, the space of smooth functions $a(z, \zeta, h)$, defined for $h \in\left(0, h_{0}\right]$ for some $h_{0}>0$, that satisfy the following property: for all $\alpha, \beta$ multi-indices, there exists $C_{\alpha, \beta} \geq 0$, such that

$$
\left|\partial_{z}^{\alpha} \partial_{\zeta}^{\beta} a(z, \zeta, h)\right| \leq C_{\alpha, \beta}\langle\zeta\rangle^{m-|\beta|}, \quad z \in \mathbf{R}^{n+1}, \zeta \in \mathbf{R}^{n+1}, h \in\left(0, h_{0}\right] .
$$

Then, for all sequences $a_{m-j} \in S^{m-j}, j \in \mathbf{N}$, there exists a symbol $a \in S^{m}$ such that $a \sim \sum_{j} h^{j} a_{m-j}$, in the sense that $a-\sum_{j<N} h^{j} a_{m-j} \in h^{N} S^{m-N}$ (see for instance [Mar02, Proposition 2.3.2] or [Hör85b, Proposition 18.1.3]), with $a_{m}$ as principal symbol. We define $\Psi^{m}$ as the space of $\psi \operatorname{DOs} A=\operatorname{Op}(a)$, for $a \in S^{m}$, formally defined by

$$
A u(z)=(2 \pi h)^{-(n+1)} \iint e^{i\langle z-t, \zeta\rangle / h} a(z, \zeta, h) u(t) d t d \zeta, \quad u \in \mathscr{P}^{\prime}\left(\mathbf{R}^{n+1}\right)
$$


We shall denote the principal symbol $a_{m}$ by $\sigma(A)$. We shall use techniques of pseudodifferential calculus in this article, such as construction of parametrices, composition formula, formula for the symbol of the adjoint operator, etc. We refer the reader to [Tay81, Hör85b, Mar02]. In the main text the variable $z$ will be $\left(x_{0}, x\right)$ and $\zeta=\left(\xi_{0}, \xi\right)$.

We now introduce tangential symbols and associated operators. We set $z=\left(z^{\prime}, z_{n}\right), z^{\prime}=\left(z_{0}, \ldots, z_{n-1}\right)$ and $\zeta^{\prime}=\left(\zeta_{0}, \ldots, \zeta_{n-1}\right)$ accordingly. We denote by $S_{\mathcal{T}}^{m}\left(\mathbf{R}^{n+1} \times \mathbf{R}^{n}\right), S_{\mathcal{T}}^{m}$ for short, the space of smooth functions $b\left(z, \zeta^{\prime}, h\right)$, defined for $h \in\left(0, h_{0}\right]$ for some $h_{0}>0$, that satisfy the following property: for all $\alpha, \beta$ multi-indices, there exists $C_{\alpha, \beta} \geq 0$, such that

$$
\left|\partial_{z}^{\alpha} \partial_{\zeta^{\prime}}^{\beta} b\left(z, \zeta^{\prime}, h\right)\right| \leq C_{\alpha, \beta}\left\langle\zeta^{\prime}\right\rangle^{m-|\beta|}, \quad z \in \mathbf{R}^{n+1}, \zeta^{\prime} \in \mathbf{R}^{n}, h \in\left(0, h_{0}\right] .
$$

As above, for all sequences $b_{m-j} \in S_{\mathcal{T}}^{m-j}, j \in \mathbf{N}$, there exists a symbol $b \in S_{\mathcal{T}}^{m}$ such that $b \sim \sum_{j} h^{j} b_{m-j}$, in the sense that $b-\sum_{j<N} h^{j} b_{m-j} \in h^{N} S_{\mathcal{T}}^{m-N}$, with $b_{m}$ as principal symbol. We define $\Psi_{\mathcal{T}}^{m}$ as the space of tangential $\psi \mathrm{DOs} B=\mathrm{op}(b)$ (observe the notation we adopt is different from above to avoid confusion), for $b \in S_{\mathcal{T}}^{m}$, formally defined by

$$
B u(z)=(2 \pi h)^{-n} \iint e^{i\left\langle z^{\prime}-t^{\prime}, \zeta^{\prime}\right\rangle / h} b\left(z, \zeta^{\prime}, h\right) u\left(t^{\prime}, z_{n}\right) d t^{\prime} d \zeta^{\prime}, \quad u \in \mathscr{P}^{\prime}\left(\mathbf{R}^{n+1}\right) .
$$

We shall also denote the principal symbol $b_{m}$ by $\sigma(B)$. In the case where the symbol is polynomial in $\zeta^{\prime}$ and $h$, we shall denote the space of associated tangential differential operators by $\mathscr{D}_{\mathcal{T}}^{m}$. We shall denote by $\Lambda^{s}$ the tangential $\psi \mathrm{DO}$ whose symbol is $\left\langle\zeta^{\prime}\right\rangle^{s}$. The composition formula for tangential symbols, $b \in S_{\mathcal{T}}^{m}$, $b^{\prime} \in S_{\mathcal{T}}^{m^{\prime}}$, is given by

$$
\begin{aligned}
& \left(b \#_{\mathcal{T}} b^{\prime}\right)\left(z, \zeta^{\prime}\right)=(2 \pi h)^{-n} \iint e^{\left.-i\left\langle t^{\prime}, \tau^{\prime}\right\rangle\right) / h} b\left(z, \zeta^{\prime}+\tau^{\prime}, h\right) b^{\prime}\left(z^{\prime}+t^{\prime}, z_{n}, \zeta^{\prime}, h\right) d t^{\prime} d \tau^{\prime} \\
& =\sum_{|\alpha| \leq M} \frac{(-i h)^{|\alpha|}}{\alpha !} \partial_{\zeta^{\prime}}^{\alpha} b\left(z, \zeta^{\prime}, h\right) \partial_{z^{\prime}}^{\alpha} b^{\prime}\left(z, \zeta^{\prime}, h\right) \\
& +\frac{(-i h)^{M+1}}{(2 \pi h)^{n}} \sum_{|\alpha|=M+1} \int_{0}^{1} \frac{(M+1)(1-s)^{M}}{\alpha !} \iint e^{\left.-i\left\langle t^{\prime}, \tau^{\prime}\right\rangle\right) / h} \partial_{\zeta^{\prime}}^{\alpha} b\left(z, \zeta^{\prime}+\tau^{\prime}, h\right) \partial_{z^{\prime}}^{\alpha} b^{\prime}\left(z^{\prime}+s t^{\prime}, z_{n}, \zeta^{\prime}, h\right) d t^{\prime} d \tau^{\prime} d s,
\end{aligned}
$$

and yields a tangential symbol in $S_{\mathcal{T}}^{m+m^{\prime}}$. In the main text the variable $z$ will be $\left(x_{0}, x^{\prime}, x_{n}\right)$ and $\zeta^{\prime}=\left(\xi_{0}, \xi^{\prime}\right)$.

Following [LR95, LR97], we shall denote by $(.,)_{0}$ the inner product for functions defined on $\left\{x_{n}=0\right\}$, i.e., $(f, g)_{0}:=\iint f\left(x_{0}, x^{\prime}\right) \bar{g}\left(x_{0}, x^{\prime}\right) d x_{0} d x^{\prime}$. The induced norm is denoted by $|\cdot|_{0}$, i.e., $|f|_{0}^{2}=(f, f)_{0}$. For $s \in \mathbf{R}$ we introduce $|f|_{s}:=\left|\Lambda^{s} f\right|_{0}$.

The outline of the article is as follows. In section 2, we prove the announced local Carleman estimate at the interface for the elliptic operator $A$. In Section 3, we prove the interpolation inequality that implies (1.2). The controllability result then follows from [LR95].

\section{Local Carleman estimate at the interface}

In the neighborhood of a point $\left(y_{0}, y\right)$ of $\left(0, X_{0}\right) \times S$, we denote by $x_{n}$ the variable that is normal to the interface $S$ and by $x^{\prime}$ the remaining spacial variables, i.e., $x=\left(x^{\prime}, x_{n}\right)$. In particular $y=\left(y^{\prime}, 0\right)$. The interface 
is now given by $S=\left\{x ; x_{n}=0\right\}$. The transmission conditions at the interface we shall consider are

$$
\forall x_{0}, x^{\prime},\left.w\right|_{x_{n}=0^{-}}=\left.w\right|_{x_{n}=0^{+}}+\theta,\left.\quad c \partial_{x_{n}} w\right|_{x_{n}=0^{-}}=\left.c \partial_{x_{n}} w\right|_{x_{n}=0^{+}}+\Theta
$$

i.e., the continuity of $w$ at the interface as well as the continuity of the normal flux, modulo some error terms $\theta$ and $\Theta$. It should be noted that for a function satisfying these transmission conditions we may not have $A w$ in $L^{2}$ in the neighborhood of $\left(y_{0}, y\right)$. It will however be in $L^{2}$ on both sides of the interface. Error terms like $\theta$ and $\Theta$ will be useful in Section 3 where the Carleman estimate proven in this section is used to achieve the null controllability result of Theorem 1.1 .

In a sufficiently small neighborhood $V \subset \mathbf{R}^{n}$ of $\left(y_{0}, y\right)$, we place ourselves in normal geodesic coordinates (w.r.t. to the spacial variables $x$ ). For convenience, we shall take the neighborhood $V$ of the form $\left(y_{0}-\right.$ $\left.\varepsilon, y_{0}+\varepsilon\right) \times V_{y^{\prime}} \times(-\varepsilon, \varepsilon)$, where $V_{y^{\prime}}$ is a sufficiently small neighborhood of $y^{\prime}$. In such coordinate system, the principal part of the differential operator $A$ takes the following form [Hör85b, Appendix C.5] on both sides of the interface:

$$
A_{2}=-\partial_{x_{0}}^{2}-c(x)\left(\partial_{x_{n}}^{2}-r\left(x, \partial_{x^{\prime}} / i\right)\right)
$$

with $r\left(x, \xi^{\prime}\right)$ a $x_{n}$-family of second-order polynomials in $\xi^{\prime}$ that satisfy

$$
r\left(x, \xi^{\prime}\right) \in \mathbf{R}, \quad \text { and } \quad C_{1}\left|\xi^{\prime}\right|^{2} \leq r\left(x, \xi^{\prime}\right) \leq C_{2}\left|\xi^{\prime}\right|^{2}, \quad x \in V_{y^{\prime}} \times(-\varepsilon, \varepsilon), \xi^{\prime} \in \mathbf{R}^{n-1},
$$

for some $0<C_{1} \leq C_{2}<\infty$. Note that the transmission conditions (TC) remain unchanged in this change of variables.

We set

$$
\begin{aligned}
& \mathbf{R}_{-}^{n+1}=\left\{\left(x_{0}, x\right), x_{n}<0\right\}, \quad \overline{\mathbf{R}}_{-}^{n+1}=\left\{\left(x_{0}, x\right), x_{n} \leq 0\right\}, \quad \mathbf{R}_{+}^{n+1}=\left\{\left(x_{0}, x\right), x_{n}>0\right\}, \quad \overline{\mathbf{R}}_{+}^{n+1}=\left\{\left(x_{0}, x\right), x_{n} \geq 0\right\}, \\
& V^{g}=V \cap \mathbf{R}_{-}^{n+1}, \quad V^{d}=V \cap \mathbf{R}_{+}^{n+1} .
\end{aligned}
$$

For a compact set $K$ of $V$ we set $K^{g}=\left\{\left(x_{0}, x\right) \in K, x_{n} \leq 0\right\}$ and $K^{d}=\left\{\left(x_{0}, x\right) \in K, x_{n} \geq 0\right\}$. We then denote by $\mathscr{C}_{c}^{\infty}\left(K^{g}\right)\left(\right.$ resp. $\mathscr{C}_{c}^{\infty}\left(K^{d}\right)$ ) the space of functions that are $\mathscr{C}^{\infty}$ in $\overline{\mathbf{R}}_{-}^{n+1}$ (resp. $\overline{\mathbf{R}}_{+}^{n+1}$ ) with support in $K^{g}$ (resp. $\left.K^{d}\right)$.

We let $\varphi$ be a (weight) function in all variables. We shall "observe" the solution of the elliptic equation $A w=f$ on the side $x_{n}>0$ and thus choose $\partial_{x_{n}} \varphi\left(x_{0}, x^{\prime}, x_{n}=0^{ \pm}\right)>0$. We shall consider three cases in order to treat the general case:

Case 1: $c\left(y^{\prime}, y_{n}=0^{-}\right)<c\left(y^{\prime}, y_{n}=0^{+}\right)$,

Case 2: $c\left(y^{\prime}, y_{n}=0^{-}\right)=c\left(y^{\prime}, y_{n}=0^{+}\right)$,

Case 3: $c\left(y^{\prime}, y_{n}=0^{-}\right)>c\left(y^{\prime}, y_{n}=0^{+}\right)$.

Recall that Case 3 is the case for which controllability and global Carleman estimates were obtained in [DOP02]. 
On both sides of $S$ we define $A_{\varphi}=h^{2} e^{\varphi / h} A_{2} e^{-\varphi / h}$. Considered as a semi-classical differential operator we denote by $a_{\varphi}$ its principal symbol, which is given by

$$
a_{\varphi}=\left(\xi_{0}+i \partial_{x_{0}} \varphi\right)^{2}+c(x)\left(\left(\xi_{n}+i \partial_{x_{n}} \varphi\right)^{2}+r\left(x, \xi^{\prime}+i \partial_{x^{\prime}} \varphi\right)\right) .
$$

We make the following assumption.

Assumption 2.1. The weight function $\varphi\left(x_{0}, x\right)$ is in $\mathscr{C}(\bar{V})$ and $\left.\varphi\right|_{\overline{\mathbf{R}}_{\mp}^{n+1}} \in \mathscr{C}^{\infty}\left(\overline{V^{g / d}}\right)$ and satisfies $\left|\nabla_{\left(x_{0}, x\right)} \varphi\right|>0$ in $\bar{V}$. We assume

$$
\forall x_{0}, x^{\prime}, \quad \partial_{x_{n}} \varphi\left(x_{0}, x^{\prime}, x_{n}=0^{ \pm}\right)>0, \quad \partial_{x_{n}} \varphi\left(x_{0}, x^{\prime}, x_{n}=0^{+}\right)-\partial_{x_{n}} \varphi\left(x_{0}, x^{\prime}, x_{n}=0^{-}\right) \geq C>0,
$$

$$
\left(c \partial_{x_{n}} \varphi\right)\left(x_{0}, x^{\prime}, x_{n}=0^{+}\right)-\left(c \partial_{x_{n}} \varphi\right)\left(x_{0}, x^{\prime}, x_{n}=0^{-}\right) \geq 0 .
$$

The function $\varphi$ satisfies the sub-ellipticity condition

$$
\forall\left(x_{0}, x, \xi_{0}, \xi\right) \in \overline{V^{g / d}} \times \mathbf{R}^{n+1}, \quad a_{\varphi}\left(x_{0}, x, \xi_{0}, \xi\right)=0 \Rightarrow\left\{\operatorname{Re} a_{\varphi}, \operatorname{Im} a_{\varphi}\right\}(x, \xi)>0 .
$$

Case 1: The neighborhood $V$ is chosen sufficiently small such that

$$
c\left(x^{\prime}, x_{n}=0^{+}\right)-c\left(x^{\prime}, x_{n}=0^{-}\right) \geq C>0, x^{\prime} \in \overline{V_{y^{\prime}}} .
$$

Moreover we assume

$$
\begin{aligned}
& \forall x_{0}, x^{\prime}, \quad\left(\partial_{x_{n}} \varphi\left(x_{0}, x^{\prime}, x_{n}=0^{+}\right)\right)^{2}-\left(\partial_{x_{n}} \varphi\left(x_{0}, x^{\prime}, x_{n}=0^{-}\right)\right)^{2} \\
&-\left(\partial_{x_{0}} \varphi\left(x_{0}, x^{\prime}, x_{n}=0\right)\right)^{2}\left(\frac{1}{c\left(x^{\prime}, x_{n}=0^{-}\right)}-\frac{1}{c\left(x^{\prime}, x_{n}=0^{+}\right)}\right) \geq C>0 .
\end{aligned}
$$

Case 2: The neighborhood $V$ is chosen sufficiently small such that $\left|c\left(x^{\prime}, x_{n}=0^{-}\right)-c\left(x^{\prime}, x_{n}=0^{+}\right)\right|$is itself sufficiently small.

Case 3: The neighborhood $V$ is chosen sufficiently small such that

$$
c\left(x^{\prime}, x_{n}=0^{+}\right)-c\left(x^{\prime}, x_{n}=0^{-}\right) \leq-C<0, x^{\prime} \in \overline{V_{y^{\prime}}} .
$$

Moreover we assume

$$
\begin{aligned}
\forall x_{0}, x^{\prime}, \quad \frac{\left(c\left(x^{\prime}, x_{n}=0^{+}\right)\right)^{2}}{c\left(x^{\prime}, x_{n}=0^{-}\right)}\left(\partial _ { x _ { n } } \varphi \left(x_{0}, x^{\prime}, x_{n}\right.\right. & \left.\left.=0^{+}\right)\right)^{2} \\
& -c\left(x^{\prime}, x_{n}=0^{-}\right) r\left(x^{\prime}, x_{n}=0, \partial_{x^{\prime}} \varphi\left(x_{0}, x^{\prime}, x_{n}=0\right)\right) \geq K,
\end{aligned}
$$

where $K$ is some positive constant and

$$
\begin{aligned}
& \forall x_{0}, x^{\prime}, \quad C_{1}\left(1-\frac{c\left(x^{\prime}, x_{n}=0^{+}\right)}{c\left(x^{\prime}, x_{n}=0^{-}\right)}\right)\left(\frac{1}{\left(\left(c \partial_{x_{n}} \varphi\right)\left(x_{0}, x^{\prime}, x_{n}=0^{-}\right)\right)^{2}}-\frac{1}{\left(\left(c \partial_{x_{n}} \varphi\right)\left(x_{0}, x^{\prime}, x_{n}=0^{+}\right)\right)^{2}}\right) \\
& -\left.C_{2}^{2}\left(\partial_{x_{0}} \varphi\right)^{2}\left(x_{0}, x^{\prime}, x_{n}=0\right)\left(\frac{1}{\left(c\left(\partial_{x_{n}} \varphi\right)^{2}\right)\left(x_{0}, x^{\prime}, x_{n}=0^{-}\right)}-\frac{1}{\left(c\left(\partial_{x_{n}} \varphi\right)^{2}\right)\left(x_{0}, x^{\prime}, x_{n}=0^{+}\right)}\right)^{2}\right|_{x_{n}=0^{+}} \geq 0
\end{aligned}
$$

where $C_{1}$ and $C_{2}$ are the constants in (2.1). 
Note that $\varphi$ is chosen continuous across the interface. In particular, we have

$$
\left.\partial_{x_{0}} \varphi\right|_{x_{n}=0^{-}}=\left.\partial_{x_{0}} \varphi\right|_{x_{n}=0^{+}},\left.\quad \partial_{x^{\prime}} \varphi\right|_{x_{n}=0^{-}}=\left.\partial_{x^{\prime}} \varphi\right|_{x_{n}=0^{+}},
$$

which we shall simply write $\left.\partial_{x_{0}} \varphi\right|_{x_{n}=0^{+}}$and $\left.\partial_{x^{\prime}} \varphi\right|_{x_{n}=0^{+}}$respectively in the sequel.

The conditions we impose on the weight function $\varphi$ will make sense in the course of the proof of Proposition 2.7 below. In Section 3 we shall construct a weight function that satisfies the properties listed in Assumption 2.1 .

From the assumption made on the weight function $\varphi$ we shall obtain the following local Carleman estimate.

Theorem 2.2. Let $K$ be a compact subset of $V$. Let the coefficient $c(x)$ satisfy Cases 1,2 or 3 . With the weight function $\varphi$ satisfying Assumption 2.1 in $\bar{V}$, there exist $C>0$ and $h_{0}>0$ such that

$$
\begin{aligned}
h\left\|e^{\varphi / h} w\right\|_{0}^{2}+h^{3}\left\|e^{\varphi / h} \nabla_{x_{0}, x} w\right\|_{0}^{2}+\left.h\left|e^{\varphi / h} w\right|_{x_{n}=0^{ \pm}}\right|_{0} ^{2}+\left.h^{3}\left|e^{\varphi / h} \partial_{x_{0}, x^{\prime}} w\right|_{x_{n}=0^{ \pm}}\right|_{0} ^{2}+\left.h^{3}\left|e^{\varphi / h} \partial_{x_{n}} w\right|_{x_{n}=0^{ \pm}}\right|_{0} ^{2} \\
\leq C\left(h^{4}\left\|e^{\varphi / h} f\right\|_{0}^{2}+h\left|e^{\varphi / h} \theta\right|_{0}^{2}+h^{3}\left|e^{\varphi / h} \partial_{x_{0}, x^{\prime}} \theta\right|_{0}^{2}+h^{3}\left|e^{\varphi / h} \Theta\right|_{0}^{2}\right), \quad 0<h \leq h_{0},
\end{aligned}
$$

for $w$ satisfying (TC), $\left.w\right|_{\overline{\mathbf{R}}_{\mp}^{n+1}} \in \mathscr{C}_{c}^{\infty}\left(K^{g / d}\right)$ and where $f=A_{2} w$ in $V \backslash S$.

Remark 2.3. This Carleman estimate yields the same estimate for the operator $A$ making use of the insensitivity of such estimates to changes of variables and to additional lower-order terms.

The remainder of this section is devoted to the proof of Theorem 2.2.

2.1. Preliminaries. We assume that the function $w$ satisfies (TC) and $A_{2} w=f$. Following [Bel03], we shall consider the transmission problem as a system of two equations on $V^{d}$ coupled at the boundary $x_{n}=0^{+}$. We thus make the change of variables $x_{n}$ to $-x_{n}$ in $V^{g}$. This yields the following system in $V^{d}$ :

$$
\left\{\begin{array}{l}
\left(-\frac{1}{c^{g}(x)} \partial_{x_{0}}^{2}-\left(\partial_{x_{n}}^{2}-r^{g}\left(x, \partial_{x^{\prime}} / i\right)\right)\right) w^{g}=F^{g}=\frac{1}{c^{g}(x)} f^{g}, \\
\left(-\frac{1}{c^{d}(x)} \partial_{x_{0}}^{2}-\left(\partial_{x_{n}}^{2}-r^{d}\left(x, \partial_{x^{\prime}} / i\right)\right)\right) w^{d}=F^{d}=\frac{1}{c^{d}(x)} f^{d},
\end{array}\right.
$$

with

$$
\left.w^{g}\right|_{x_{n}=0^{+}}=\left.w^{d}\right|_{x_{n}=0^{+}}+\theta,\left.\quad c^{g} \partial_{x_{n}} w^{g}\right|_{x_{n}=0^{+}}+\left.c^{d} \partial_{x_{n}} w^{d}\right|_{x_{n}=0^{+}}=\Theta,
$$

where for a function $\psi$ defined in $V$, we set $\psi^{d}:=\left.\psi\right|_{V^{d}}$ and $\psi^{g}\left(x^{\prime}, x_{n}\right)=\psi\left(x^{\prime},-x_{n}\right)$ for $x_{n}>0$. In particular, we have $r^{g}\left(x, \partial_{x^{\prime}} / i\right)=r\left(x^{\prime},-x_{n}, \partial_{x^{\prime}} / i\right)$, and $r^{d}\left(x, \partial_{x^{\prime}} / i\right)=r\left(x, \partial_{x^{\prime}} / i\right)$ for $x_{n}>0$. If there is no possible confusion, we shall now write $\psi={ }^{t}\left(\psi^{g}, \psi^{d}\right)$. From Assumption 2.1 we have

$$
\partial_{x_{n}} \varphi^{g}\left(x_{0}, x^{\prime}, x_{n}=0\right)<0, \quad \partial_{x_{n}} \varphi^{d}\left(x_{0}, x^{\prime}, x_{n}=0\right)>0,
$$

and

$$
c^{g} \partial_{x_{n}} \varphi^{g}\left(x_{0}, x^{\prime}, x_{n}=0\right)+c^{d} \partial_{x_{n}} \varphi^{d}\left(x_{0}, x^{\prime}, x_{n}=0\right) \geq 0 .
$$

Observe also that condition (2.3) is preserved since $\left\{\operatorname{Re} a_{\varphi}, \operatorname{Im} a_{\varphi}\right\}$ is invariant under a change of variables [Hör63, Section 8.1, page 186]. 
We denote by $p^{g / d}$ the symbols of the operators acting on $w^{g / d}$ in (2.8). We set $P\left(x_{0}, x, D_{x_{0}}, D_{x}\right):=$ $\operatorname{Op}\left(\operatorname{diag}\left(p^{g}, p^{d}\right)\right)$ and $\Phi:=\operatorname{diag}\left(\varphi^{g}, \varphi^{d}\right)$. We set $v={ }^{t}\left(v^{g}, v^{d}\right)$. For $v=e^{\Phi / h} w$, the entries of $v$ satisfy the following boundary condition

$$
\left.v^{g}\right|_{x_{n}=0^{+}}=\left.v^{d}\right|_{x_{n}=0^{+}}+\theta_{\varphi},\left.\quad c^{g}\left(D_{x_{n}}+i \partial_{x_{n}} \varphi^{g}\right) v^{g}\right|_{x_{n}=0^{+}}+\left.c^{d}\left(D_{x_{n}}+i \partial_{x_{n}} \varphi^{d}\right) v^{d}\right|_{x_{n}=0^{+}}=\Theta_{\varphi},
$$

where

$$
\theta_{\varphi}=\left.e^{\varphi / h}\right|_{x_{n}=0^{+}} \theta \quad \text { and } \quad \Theta_{\varphi}=\left.\frac{h}{i} e^{\varphi / h}\right|_{x_{n}=0^{+}} \Theta .
$$

We define the following conjugated operator $P_{\varphi}=h^{2} e^{\Phi / h} P e^{-\Phi / h}$, which we shall, in the sequel, treat as a second-order semi-classical differential operator, with $h$ as the small parameter. The principal symbol of $P_{\varphi}$ is given by

$$
p_{\varphi}\left(x_{0}, x, \xi_{0}, \xi^{\prime}, \xi_{n}\right)=\operatorname{diag}\left(p_{\varphi}^{g}\left(x_{0}, x, \xi_{0}, \xi^{\prime}, \xi_{n}\right), p_{\varphi}^{d}\left(x_{0}, x, \xi_{0}, \xi^{\prime}, \xi_{n}\right)\right)
$$

with

$$
p_{\varphi}^{g / d}\left(x_{0}, x, \xi_{0}, \xi^{\prime}, \xi_{n}\right)=\frac{1}{c^{g / d}}\left(\xi_{0}+i \partial_{x_{0}} \varphi^{g / d}\right)^{2}+\left(\xi_{n}+i \partial_{x_{n}} \varphi^{g / d}\right)^{2}+r^{g / d}\left(x, \xi^{\prime}+i \partial_{x^{\prime}} \varphi^{g / d}\right) .
$$

For the sake of concision, we shall often omit the time and spacial variables in the functions $c^{g / d}$ and $\varphi^{g / d}$, as we have just done, when there is no possible confusion. Separating the real and imaginary parts of the principal symbol, we write $p_{\varphi}^{g / d}=\tilde{q}_{2}^{g / d}+i \tilde{q}_{1}^{g / d}$, and following [LR95] we set

$$
\tilde{q}_{2}^{g / d}=\xi_{n}^{2}+q_{2}^{g / d}, \quad \tilde{q}_{1}^{g / d}=2 \partial_{x_{n}} \varphi^{g / d} \xi_{n}+2 q_{1}^{g / d},
$$

with

$$
\begin{aligned}
& q_{2}^{g / d}\left(x_{0}, x, \xi_{0}, \xi^{\prime}\right)=-\left(\partial_{x_{n}} \varphi^{g / d}\right)^{2}+\frac{1}{c^{g / d}}\left(\xi_{0}^{2}-\left(\partial_{x_{0}} \varphi^{g / d}\right)^{2}\right)+r^{g / d}\left(x, \xi^{\prime}\right)-r^{g / d}\left(x, \partial_{x^{\prime}} \varphi^{g / d}\right), \\
& q_{1}^{g / d}\left(x_{0}, x, \xi_{0}, \xi^{\prime}\right)=\frac{1}{c^{g / d}} \partial_{x_{0}} \varphi^{g / d} \xi_{0}+\tilde{r}^{g / d}\left(x, \xi^{\prime}, \partial_{x^{\prime}} \varphi^{g / d}\right),
\end{aligned}
$$

where $\tilde{r}^{g / d}\left(x, \xi^{\prime}, \eta^{\prime}\right)$ are the symmetric bilinear forms in $\xi^{\prime}, \eta^{\prime}$ associated to the real quadratic forms $r^{g / d}\left(x, \xi^{\prime}\right)$.

2.2. Signs of the imaginary part of the two roots of $\boldsymbol{p}_{\varphi}^{g / d}$. At $x_{n}=0^{+}$, the polynomials (in $\left.\xi_{n}\right) p_{\varphi}^{g / d}\left(x_{0}, x, \xi_{0}, \xi^{\prime}, \xi_{n}\right)$ have two complex roots. Depending on the signs of the imaginary parts of the two roots of the two polynomials, we shall adopt different strategies for the proof of partial Carleman estimates. By "partial" we actually mean that the resulting estimate will only hold in some microlocal region. Once collected together, the partial estimates will yield the result of Theorem 2.2.

Following [LR97], we set

$$
\mu^{g / d}\left(x_{0}, x, \xi_{0}, \xi^{\prime}\right):=q_{2}^{g / d}\left(x_{0}, x, \xi_{0}, \xi^{\prime}\right)+\frac{\left(q_{1}^{g / d}\left(x_{0}, x, \xi_{0}, \xi^{\prime}\right)\right)^{2}}{\left(\partial_{x_{n}} \varphi^{g / d}\right)^{2}},
$$


and define

$$
\begin{aligned}
& \mathscr{E}^{\% /,+}:=\left\{\left(x_{0}, x, \xi_{0}, \xi^{\prime}\right) \in V^{d} \times \mathbf{R}^{n} ; \mu^{g / d}\left(x_{0}, x, \xi_{0}, \xi^{\prime}\right)>0\right\}, \\
& \mathscr{E}^{g / d,-}:=\left\{\left(x_{0}, x, \xi_{0}, \xi^{\prime}\right) \in V^{d} \times \mathbf{R}^{n} ; \mu^{g / d}\left(x_{0}, x, \xi_{0}, \xi^{\prime}\right)<0\right\}, \\
& \mathscr{J}^{g / d}:=\left\{\left(x_{0}, x, \xi_{0}, \xi^{\prime}\right) \in V^{d} \times \mathbf{R}^{n} ; \mu^{g / d}\left(x_{0}, x, \xi_{0}, \xi^{\prime}\right)=0\right\} .
\end{aligned}
$$

Remark 2.4. The regions $\mathscr{E}^{\frac{g}{d},-}$ and $\mathcal{Z}^{g / d}$ are bounded. Hence, for $\left|\left(\xi_{0}, \xi^{\prime}\right)\right|$ sufficiently large, say $\left|\left(\xi_{0}, \xi^{\prime}\right)\right|>$ $R$, then $\left(x_{0}, x, \xi_{0}, \xi^{\prime}\right) \in \mathscr{E}^{g,+} \cap \mathscr{E}^{d,+}$, with $\operatorname{dist}\left(\left(x_{0}, x, \xi_{0}, \xi^{\prime}\right), \mathscr{J}^{g / d}\right) \geq C>0$.

The following lemma is proven in [LR97, proof of Lemma 3].

Lemma 2.5. In the region $\mathscr{E}^{\mathscr{\%} /,+}$, the polynomials $p_{\varphi}^{g / d}$ have two distinct roots $\rho^{g / d,+}$ and $\rho^{g / d,-}$ that satisfy $\operatorname{Im} \rho^{g / d,+}>0$ and $\operatorname{Im} \rho^{g / d,-}<0$. In the region $\mathscr{E}^{\% /,-}$, the imaginary parts of the two roots have the same sign as that of $-\partial_{x_{n}} \varphi^{g / d}$. In $\mathcal{Z}^{g / d}$, one of the roots is real.

Hence, for the polynomial $p_{\varphi}^{d}$, for $\left|\left(\xi_{0}, \xi^{\prime}\right)\right|>R$, there are two roots, $\rho^{d,+}$ and $\rho^{d,-}$ with $\operatorname{Im} \rho^{d,+}>0$ and $\operatorname{Im} \rho^{d,-}<0$. As the value of $\mu^{d}$ decreases, the root $\rho^{d,+}$ moves towards the real axis, and crosses it in the region $\mathcal{Z}^{d}$. In the region $\mathscr{E}^{d,-}$ the two roots both have negative imaginary parts.

For the polynomial $p_{\varphi}^{g}$, for $\left|\left(\xi_{0}, \xi^{\prime}\right)\right|>R$, there are two roots, $\rho^{g,+}$ and $\rho^{g,-}$ with $\operatorname{Im} \rho^{g,+}>0$ and $\operatorname{Im} \rho^{g,-}<$ 0 . As the value of $\mu^{g}$ decreases, the root $\rho^{g,-}$ moves towards the real axis, and crosses it in the region $\mathcal{Z}^{g}$. In the region $\mathscr{E}^{g}$,- the two roots both have positive imaginary parts. The "motion" of the roots of $p_{\varphi}^{g}$ and $p_{\varphi}^{d}$ is illustrated in Figure 1.

Remark 2.6. From the proof of Lemma 3 in [LR97], we see that $\mu^{g / d} \geq C>0$ is equivalent to having $\operatorname{Im} \rho^{g / d,+} \geq C^{\prime}>0$ and $\operatorname{Im} \rho^{g / d,-} \leq-C^{\prime}$.

With the choice of weight function $\varphi$ made in Assumption 2.1 we have the following proposition.

Proposition 2.7. The properties of the weight function $\varphi$ imply $\mathscr{E}^{d,+} \subset \mathscr{E}^{g,+}$, and $\operatorname{dist}\left(\mathscr{E}^{d,+}, \mathscr{Z}^{g}\right) \geq C>0$, if the neighborhood $V_{y}$ of $y$ is chosen sufficiently small.

The result of the proposition implies that the root $\rho^{d,+}$ crosses the real axis before the root $\rho^{g,-}$ does, as $\mu^{d}$ decreases from positive to negative values. This is illustrated in Figure 1. We enforce this root configuration because of the techniques we shall use to prove partial Carleman estimates.

In the case where the roots of the polynomial are separated by the real axis, or in the case where they are both in the lower open half plane, we can apply the Calderón-projector technique to the associated differential operator. The first case occurs for $P_{\varphi}^{g / d}$ in regions $\mathscr{E}^{\% /,+}$. The second case can only occur for $P_{\varphi}^{d}$ in the region $\mathscr{E}^{d,-}$. In such regions, the Calderón-projector technique in fact yields an additional boundary condition at $x_{n}=0^{+}$.

In fact, the choice of weight function $\varphi$ we have made excludes the situation in which $\operatorname{Im} \rho^{g, \pm}>0$ and the root $\rho^{d,+}$ may cross the real axis. In such a case, the Calderón projector technique cannot be used for 


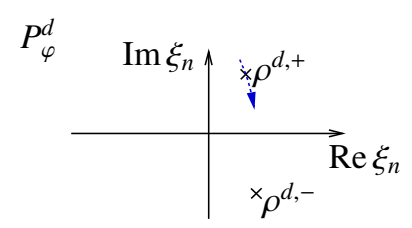

$P_{\varphi}^{g}$

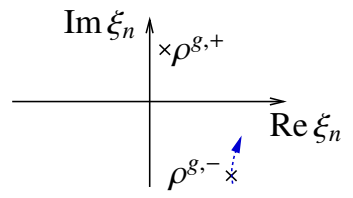

(a) Root configuration in $\mathscr{E}^{d,+}, \mu^{d}>0$;

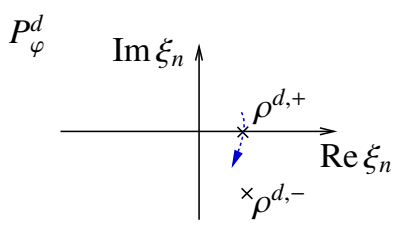

$P_{\varphi}^{g}$

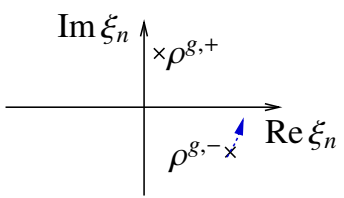

(b) Root configuration in $\mathscr{J}^{d}, \mu^{d}=0$;

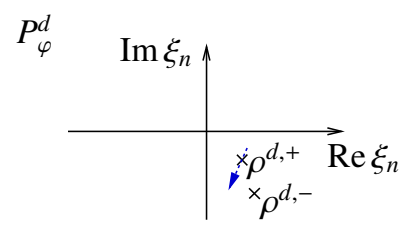

$P_{\varphi}^{g}$

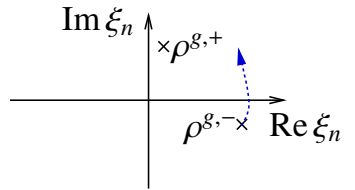

(c) Root configuration in $\mathscr{E}^{d,-}, \mu^{d}<0$.

Figure 1: The root $\rho^{d,+}$ crosses the real axis before the root $\rho^{g,-}$ does, as $\mu^{d}$ decreases.

$P_{\varphi}^{g}$ or $P_{\varphi}^{d}$. The classical Carleman technique then yields a quadratic form for the traces of $v$ and its normal derivative $D_{x_{n}} v$ which is of unknown or negative sign, which prevents the derivation of a proper Carleman type estimate.

Proof of Proposition 2.7. The result is clear in the case $\left|\left(\xi_{0}, \xi^{\prime}\right)\right|>R$ by Remark 2.4. We shall thus only consider the case $\left|\left(\xi_{0}, \xi^{\prime}\right)\right| \leq R$. We set $W=\left\{\left(x_{0}, x^{\prime}, \xi_{0}, \xi^{\prime}\right) \in[s-\varepsilon, s+\varepsilon] \times \bar{V}_{y^{\prime}} \times \mathbf{R}^{n} ;\left|\left(\xi_{0}, \xi^{\prime}\right)\right| \leq R\right\}$. A sufficient condition to prove the result is then

$$
\left.\mu^{g}\left(x_{0}, x, \xi_{0}, \xi^{\prime}\right)\right|_{x_{n}=0^{+}}-\left.\mu^{d}\left(x_{0}, x, \xi_{0}, \xi^{\prime}\right)\right|_{x_{n}=0^{+}} \geq C>0, \quad\left(x_{0}, x^{\prime}, \xi_{0}, \xi^{\prime}\right) \in W
$$

In fact, since $W$ is compact, by choosing $V$ sufficiently small in the $x_{n}$-direction, this inequality remains valid in $V^{d} \times \mathbf{R}^{n} \cap\left\{\left|\left(\xi_{0}, \xi^{\prime}\right)\right| \leq R\right\}$ and the result follows.

We first treat Case 1. Observing that

$$
\begin{aligned}
& \left.r^{d}\left(x, \partial_{x^{\prime}} \varphi^{d}\right)\right|_{x_{n}=0^{+}}=\left.r^{g}\left(x, \partial_{x^{\prime}} \varphi^{g}\right)\right|_{x_{n}=0^{+}}, \\
& \left.\tilde{r}\left(x, \xi^{\prime}, \partial_{x^{\prime}} \varphi\right)\right|_{x_{n}=0^{+}}:=\left.\tilde{r}^{d}\left(x, \xi^{\prime}, \partial_{x^{\prime}} \varphi^{d}\right)\right|_{x_{n}=0^{+}}=\left.\tilde{r}^{g}\left(x, \xi^{\prime}, \partial_{x^{\prime}} \varphi^{g}\right)\right|_{x_{n}=0^{+}},
\end{aligned}
$$

we obtain

$$
\begin{array}{r}
\left.\left(\mu^{g}-\mu^{d}\right)\left(x_{0}, x, \xi_{0}, \xi^{\prime}\right)\right|_{x_{n}=0^{+}}=\left(\left.\partial_{x_{n}} \varphi^{d}\right|_{x_{n}=0^{+}}\right)^{2}-\left(\left.\partial_{x_{n}} \varphi^{g}\right|_{x_{n}=0^{+}}\right)^{2}+\left(\xi_{0}^{2}-\left.\left(\left.\partial_{x_{0}} \varphi\right|_{x_{n}=0^{+}}\right)\left(\frac{1}{c^{g}}-\frac{1}{c^{d}}\right)\right|_{x_{n}=0^{+}}\right. \\
+\left(\frac{\left.\left(\left.\frac{1}{c^{g}} \xi_{0} \partial_{x_{0}} \varphi\right|_{x_{n}=0^{+}}+\tilde{r}\left(x, \xi^{\prime}, \partial_{x^{\prime}} \varphi\right)\right)\right|_{x_{n}=0^{+}}}{\left.\partial_{x_{n}} \varphi^{g}\right|_{x_{n}=0^{+}}}\right)^{2}-\left(\frac{\left.\left(\left.\frac{1}{c^{d}} \xi_{0} \partial_{x_{0}} \varphi\right|_{x_{n}=0^{+}}+\tilde{r}\left(x, \xi^{\prime}, \partial_{x^{\prime}} \varphi\right)\right)\right|_{x_{n}=0^{+}}}{\left.\partial_{x_{n}} \varphi^{d}\right|_{x_{n}=0^{+}}}\right)^{2},
\end{array}
$$


which, after expansion, we write

$$
\begin{aligned}
\left.\left(\mu^{g}-\mu^{d}\right)\left(x_{0}, x, \xi_{0}, \xi^{\prime}\right)\right|_{x_{n}=0^{+}} & =\left(\left.\partial_{x_{n}} \varphi^{d}\right|_{x_{n}=0^{+}}\right)^{2}-\left(\left.\partial_{x_{n}} \varphi^{g}\right|_{x_{n}=0^{+}}\right)^{2}-\left.\left(\left.\partial_{x_{0}} \varphi\right|_{x_{n}=0^{+}}\right)^{2}\left(\frac{1}{c^{g}}-\frac{1}{c^{d}}\right)\right|_{x_{n}=0^{+}} \\
& +\xi_{0}^{2}\left(\left.\left(\frac{1}{c^{g}}-\frac{1}{c^{d}}\right)\right|_{x_{n}=0^{+}}+\left.\left(\left.\partial_{x_{0}} \varphi\right|_{x_{n}=0^{+}}\right)^{2}\left(\frac{1}{\left(c^{g} \partial_{x_{n}} \varphi^{g}\right)^{2}}-\frac{1}{\left(c^{d} \partial_{x_{n}} \varphi^{d}\right)^{2}}\right)\right|_{x_{n}=0^{+}}\right) \\
& +\left.\left.\left(\tilde{r}\left(x, \xi^{\prime}, \partial_{x^{\prime}} \varphi\right)\right)^{2}\right|_{x_{n}=0^{+}}\left(\frac{1}{\left(\partial_{x_{n}} \varphi^{g}\right)^{2}}-\frac{1}{\left(\partial_{x_{n}} \varphi^{d}\right)^{2}}\right)\right|_{x_{n}=0^{+}} \\
& +\left.\left.2 \xi \tilde{r}\left(x, \xi^{\prime}, \partial_{x^{\prime}} \varphi\right) \partial_{x_{0}} \varphi\right|_{x_{n}=0^{+}}\left(\frac{1}{c^{g}\left(\partial_{x_{n}} \varphi^{g}\right)^{2}}-\frac{1}{c^{d}\left(\partial_{x_{n}} \varphi^{d}\right)^{2}}\right)\right|_{x_{n}=0^{+}}
\end{aligned}
$$

The first line in (2.14) is larger than some positive constant by (2.4) in Assumption 2.1-Case 1. The last three lines in (2.14) can be viewed as a quadratic form in $\xi_{0}$ and $\left.\tilde{r}\left(x, \xi^{\prime}, \partial_{x^{\prime}} \varphi\right)\right|_{x_{n}=0^{+}}$. The determinant of the associated symmetric matrix is given by

$$
\left.\left(\frac{1}{c^{g}}-\frac{1}{c^{d}}\right) \frac{1}{\left(\partial_{x_{n}} \varphi^{g}\right)^{2}\left(\partial_{x_{n}} \varphi^{d}\right)^{2}}\left(\left(\partial_{x_{n}} \varphi^{d}\right)^{2}-\left(\partial_{x_{n}} \varphi^{g}\right)^{2}-\left(\partial_{x_{0}} \varphi\right)^{2}\left(\frac{1}{c^{g}}-\frac{1}{c^{d}}\right)\right)\right|_{x_{n}=0^{+}},
$$

and is thus positive by (2.4). Since the coefficient in front of $\left.\tilde{r}\left(x, \xi^{\prime}, \partial_{x^{\prime}} \varphi\right)\right|_{x_{n}=0^{+}}$in (2.14) is itself positive by Assumption 2.1, we find that the quadratic form is nonnegative. The sufficient condition (2.13) hence follows.

We now treat Case 2. We write

$$
\begin{aligned}
& \left.\left(\mu^{g}-\mu^{d}\right)\left(x_{0}, x, \xi_{0}, \xi^{\prime}\right)\right|_{x_{n}=0^{+}}=\left(\left.\partial_{x_{n}} \varphi^{d}\right|_{x_{n}=0^{+}}\right)^{2}-\left(\left.\partial_{x_{n}} \varphi^{g}\right|_{x_{n}=0^{+}}\right)^{2} \\
& \left.\quad+\left.\left(\left.\frac{1}{c^{d}} \xi_{0} \partial_{x_{0}} \varphi\right|_{x_{n}=0^{+}}+\tilde{r}\left(x, \xi^{\prime}, \partial_{x^{\prime}} \varphi\right)\right)\right|_{x_{n}=0^{+}}\right)^{2}\left(\frac{1}{\left(\left.\partial_{x_{n}} \varphi^{g}\right|_{x_{n}=0^{+}}\right)^{2}}-\frac{1}{\left(\left.\partial_{x_{n}} \varphi^{d}\right|_{x_{n}=0^{+}}\right)^{2}}\right) \\
& \left.\quad+\left.\left(\frac{1}{c^{g}}-\frac{1}{c^{d}}\right)\right|_{x_{n}=0^{+}}\left(\xi_{0}^{2}-\left(\left.\partial_{x_{0}} \varphi\right|_{x_{n}=0^{+}}\right)^{2}+\left.\left.2 \xi_{0} \partial_{x_{0}} \varphi\right|_{x_{n}=0^{+}}\left(\tilde{r}\left(x, \xi^{\prime}, \partial_{x^{\prime}} \varphi\right)\right)\right|_{x_{n}=0^{+}}+\frac{\left.\partial_{x_{0}} \varphi\right|_{x_{n}=0^{+}} \xi_{0}}{\left.c^{d}\right|_{x_{n}=0^{+}}}\right)\right) \\
& \quad+\frac{\left.\left(\frac{1}{c^{g}}-\frac{1}{c^{d}}\right)^{2}\right|_{x_{n}=0^{+}}}{\left(\left.\partial_{x_{n}} \varphi^{g}\right|_{x_{n}=0^{+}}\right)^{2}}\left(\left.\xi_{0} \partial_{x_{0}} \varphi\right|_{x_{n}=0^{+}} \xi_{0}\right)^{2} .
\end{aligned}
$$

With $\left|\left(\xi_{0}, \xi^{\prime}\right)\right| \leq R$, we see that the last two terms in the previous expression can be made as small as desired by choosing the neighborhood $V_{y^{\prime}}$ sufficiently small, which implies $\left|c\left(x^{\prime}, x_{n}=0^{-}\right)-c\left(x^{\prime}, x_{n}=0^{+}\right)\right|$small. The sum of the first two terms in (2.15) is larger than some positive constant by the properties of $\varphi$ in Assumption 2.1, which yields the conclusion.

We finally treat Case 3. Then $\left.c^{g}\right|_{x_{n}=0^{+}}>\left.c^{d}\right|_{x_{n}=0^{+}}$. In particular, note that

$$
\left.c^{d}\left(\partial_{x_{n}} \varphi^{d}\right)^{2}\right|_{x_{n}=0^{+}} \geq\left. c^{g}\left(\partial_{x_{n}} \varphi^{g}\right)^{2}\right|_{x_{n}=0^{+}},
$$

from Assumption 2.1. Observe that in this case we have

$$
\left.c^{g} q_{2}^{g}\left(x_{0}, x, \xi_{0}, \xi^{\prime}\right)\right|_{x_{n}=0^{+}} \geq \lambda\left(x_{0}, x^{\prime}, \xi_{0}, \xi^{\prime}\right),\left.\quad c^{d} q_{2}^{d}\left(x_{0}, x, \xi_{0}, \xi^{\prime}\right)\right|_{x_{n}=0^{+}} \geq \lambda\left(x_{0}, x^{\prime}, \xi_{0}, \xi^{\prime}\right),
$$


where

$$
\lambda\left(x_{0}, x^{\prime}, \xi_{0}, \xi^{\prime}\right)=\xi_{0}^{2}-\left(\partial_{x_{0}} \varphi\right)^{2}-\left.c^{d}\left(\partial_{x_{n}} \varphi^{d}\right)^{2}\right|_{x_{n}=0^{+}}+\left.c^{d} r\left(x, \xi^{\prime}\right)\right|_{x_{n}=0^{+}}-\left.c^{g} r\left(x, \partial_{x^{\prime}} \varphi\right)\right|_{x_{n}=0^{+}} \cdot
$$

Let $K$ be the constant appearing in (2.5). In the case $\lambda\left(x_{0}, x^{\prime}, \xi_{0}, \xi^{\prime}\right)>K / 2$, then locally, for $x_{n} \geq 0,\left|x_{n}\right|$ small, this remains valid with $K / 2$ changed into $K / 4$. Locally, we thus have $\mu^{g} \geq K /\left(4 c_{\max }\right)>0$ and $\mu^{d} \geq K /\left(4 c_{\max }\right)>0$, from the definitions of $\mu^{g / d}$ in (2.12). In the region $\lambda\left(x_{0}, x^{\prime}, \xi_{0}, \xi^{\prime}\right)>K / 2$ the result is hence clear.

We now treat the region $\lambda\left(x_{0}, x^{\prime}, \xi_{0}, \xi^{\prime}\right) \leq K / 2$. By choosing the neighborhood $V$ sufficiently small, arguing as above, it is now sufficient to prove that

$$
\left.\mu^{g}\left(x_{0}, x, \xi_{0}, \xi^{\prime}\right)\right|_{x_{n}=0^{+}}-\left.\mu^{d}\left(x_{0}, x, \xi_{0}, \xi^{\prime}\right)\right|_{x_{n}=0^{+}} \geq C>0, \quad\left(x_{0}, x^{\prime}, \xi_{0}, \xi^{\prime}\right) \in \widetilde{W}
$$

where $\widetilde{W}=W \cap\left\{\left(x_{0}, x^{\prime}, \xi_{0}, \xi^{\prime}\right) ; \lambda\left(x_{0}, x^{\prime}, \xi_{0}, \xi^{\prime}\right) \leq K / 2\right\}$ which is compact. From Assumption 2.1, we observe that we have

$$
\left(\partial_{x_{n}} \varphi^{d} \mid x_{n}=0^{+}\right)^{2}-\left(\left.\partial_{x_{n}} \varphi^{g}\right|_{x_{n}=0^{+}}\right)^{2} \geq\left.\left(c^{d}\right)^{2}\left(\frac{1}{c^{d}}+\frac{1}{c^{g}}\right)\left(\frac{1}{c^{d}}-\frac{1}{c^{g}}\right)\right|_{x_{n}=0^{+}}\left(\left.\partial_{x_{n}} \varphi^{d}\right|_{x_{n}=0^{+}}\right)^{2} .
$$

With $\lambda\left(x_{0}, x^{\prime}, \xi_{0}, \xi^{\prime}\right) \leq K / 2$ in $\widetilde{W}$, we then obtain

$$
\begin{aligned}
&\left.\left(\mu^{g}-\mu^{d}\right)\left(x_{0}, x, \xi_{0}, \xi^{\prime}\right)\right|_{x_{n}=0^{+}}=Q+\left.\xi_{0}^{2}\left(\left.\partial_{x_{0}} \varphi\right|_{x_{n}=0^{+}}\right)^{2}\left(\frac{1}{\left(c^{g} \partial_{x_{n}} \varphi^{g}\right)^{2}}-\frac{1}{\left(c^{d} \partial_{x_{n}} \varphi^{d}\right)^{2}}\right)\right|_{x_{n}=0^{+}} \\
&+\left.\left.\left(\tilde{r}\left(x, \xi^{\prime}, \partial_{x^{\prime}} \varphi\right)\right)^{2}\right|_{x_{n}=0^{+}}\left(\frac{1}{\left(\partial_{x_{n}} \varphi^{g}\right)^{2}}-\frac{1}{\left(\partial_{x_{n}} \varphi^{d}\right)^{2}}\right)\right|_{x_{n}=0^{+}} \\
&+\left.\left.2 \xi \tilde{r}\left(x, \xi^{\prime}, \partial_{x^{\prime}} \varphi\right) \partial_{x_{0}} \varphi\right|_{x_{n}=0^{+}}\left(\frac{1}{c^{g}\left(\partial_{x_{n}} \varphi^{g}\right)^{2}}-\frac{1}{c^{d}\left(\partial_{x_{n}} \varphi^{d}\right)^{2}}\right)\right|_{x_{n}=0^{+}},
\end{aligned}
$$

where

$$
\begin{aligned}
Q & \geq\left.\left(\frac{1}{c^{d}}-\frac{1}{c^{g}}\right)\right|_{x_{n}=0^{+}}\left\{\left.\frac{\left(c^{d}\right)^{2}}{c^{g}}\right|_{x_{n}=0^{+}}\left(\left.\partial_{x_{n}} \varphi^{d}\right|_{x_{n}=0^{+}}\right)^{2}+\left.c^{d} r\left(x, \xi^{\prime}\right)\right|_{x_{n}=0^{+}}-\left.c^{g} r\left(x, \partial_{x^{\prime}} \varphi\right)\right|_{x_{n}=0^{+}}-K / 2\right\} \\
& \geq\left.\left(\frac{1}{c^{d}}-\frac{1}{c^{g}}\right)\right|_{x_{n}=0^{+}}\left(K / 2+\left.c^{d} r\left(x, \xi^{\prime}\right)\right|_{x_{n}=0^{+}}\right) \geq\left.\left(\frac{1}{c^{d}}-\frac{1}{c^{g}}\right)\right|_{x_{n}=0^{+}}\left(K / 2+C_{1}\left(\left.c^{d}\right|_{x_{n}=0^{+}}\right)\left|\xi^{\prime}\right|^{2}\right),
\end{aligned}
$$

by (2.5) in Assumption 2.1 and where $C_{1}$ is the uniform-ellipticity constant appearing in (2.1). As we have

$$
\left.\left|\tilde{r}\left(x, \xi^{\prime}, \partial_{x^{\prime}} \varphi\right)\right|_{x_{n}=0^{+}}\left|\leq C_{2}\right| \xi^{\prime}|| \partial_{x^{\prime}} \varphi\right|_{x_{n}=0^{+}} \mid
$$


with $C_{2}$ also appearing in (2.1), we obtain

$$
\begin{aligned}
\left.\left(\mu^{g}-\mu^{d}\right)\left(x_{0}, x, \xi_{0}, \xi^{\prime}\right)\right|_{x_{n}=0^{+}} & \geq\left.\left(\frac{1}{c^{d}}-\frac{1}{c^{g}}\right)\right|_{x_{n}=0^{+}} K / 2+\left.\xi_{0}^{2}\left(\left.\partial_{x_{0}} \varphi\right|_{x_{n}=0^{+}}\right)^{2}\left(\frac{1}{\left(c^{g} \partial_{x_{n}} \varphi^{g}\right)^{2}}-\frac{1}{\left(c^{d} \partial_{x_{n}} \varphi^{d}\right)^{2}}\right)\right|_{x_{n}=0^{+}} \\
& +\left.C_{1} c^{d}\left(\frac{1}{c^{d}}-\frac{1}{c^{g}}\right)\right|_{x_{n}=0^{+}}\left|\xi^{\prime}\right|^{2} \\
& -\left.2 C_{2}\left|\partial_{x_{0}} \varphi\right|_{x_{n}=0^{+}}|| \partial_{x^{\prime}} \varphi\right|_{x_{n}=0^{+}}|| \xi_{0}|| \xi^{\prime}\left|\left(\frac{1}{c^{g}\left(\partial_{x_{n}} \varphi^{g}\right)^{2}}-\frac{1}{c^{d}\left(\partial_{x_{n}} \varphi^{d}\right)^{2}}\right)\right|_{x_{n}=0^{+}},
\end{aligned}
$$

since the third term in the r.h.s. of (2.17) is nonnegative by Assumption 2.5. Next, we consider the last three terms in (2.18) as a quadratic form in $\left|\xi^{\prime}\right|$ and $\xi_{0}$. The coefficients associated to $\xi_{0}^{2}$ and $\left|\xi^{\prime}\right|^{2}$ are nonnegative. The result of the proposition follows if the quadratic form is nonnegative, that is, if its determinant is itself nonnegative. The determinant is given by

$$
\begin{aligned}
\left(\left.\partial_{x_{0}} \varphi\right|_{x_{n}=0^{+}}\right)^{2}\left\{\left.\left.C_{1}\left(1-\frac{c^{d}}{c^{g}}\right)\right|_{x_{n}=0^{+}}\left(\frac{1}{\left(c^{g} \partial_{x_{n}} \varphi^{g}\right)^{2}}-\frac{1}{\left(c^{d} \partial_{x_{n}} \varphi^{d}\right)^{2}}\right)\right|_{x_{n}=0^{+}}\right. & \\
& \left.-\left.C_{2}^{2}\left(\left.\partial_{x_{0}} \varphi\right|_{x_{n}=0^{+}}\right)^{2}\left(\frac{1}{c^{g}\left(\partial_{x_{n}} \varphi^{g}\right)^{2}}-\frac{1}{c^{d}\left(\partial_{x_{n}} \varphi^{d}\right)^{2}}\right)^{2}\right|_{x_{n}=0^{+}}\right\},
\end{aligned}
$$

and is nonnegative by (2.6) in Assumption 2.1-Case 3.

Remark 2.8. Because of the controllability result we aim to prove in this article, we have considered the elliptic operator $A:=-\partial_{x_{0}}^{2}-L$, with the additional variable $x_{0}$. The Carleman estimate of Theorem 2.2 also holds for the operator $\left.L=\nabla_{x} \cdot\left(c(x) \nabla_{x}\right)\right)$. In this case, we simply assume that the weight function satisfies

$$
\forall x_{0}, x^{\prime}, \quad \partial_{x_{n}} \varphi\left(x_{0}, x^{\prime}, x_{n}=0^{ \pm}\right)>0, \quad \partial_{x_{n}} \varphi\left(x_{0}, x^{\prime}, x_{n}=0^{+}\right)-\partial_{x_{n}} \varphi\left(x_{0}, x^{\prime}, x_{n}=0^{-}\right) \geq C>0 .
$$

In fact, in this case, after dividing by $c(x)$ on both sides of the interface $S$ as above, the symbols $q_{2}^{g / d}$ and $q_{1}^{g / d}$ reduce to

$$
q_{2}^{g / d}\left(x, \xi^{\prime}\right)=-\left(\partial_{x_{n}} \varphi^{g / d}\right)^{2}+r^{g / d}\left(x, \xi^{\prime}\right)-r^{g / d}\left(x, \partial_{x^{\prime}} \varphi^{g / d}\right), \quad q_{1}^{g / d}\left(x, \xi^{\prime}\right)=\tilde{r}^{g / d}\left(x, \xi^{\prime}, \partial_{x^{\prime}} \varphi^{g / d}\right) .
$$

We then have

$$
\begin{aligned}
\left.\mu^{g}\left(x, \xi^{\prime}\right)\right|_{x_{n}=0^{+}}-\left.\mu^{d}\left(x, \xi^{\prime}\right)\right|_{x_{n}=0^{+}} & =\left(\left(\left.\partial_{x_{n}} \varphi^{d}\right|_{x_{n}=0^{+}}\right)^{2}-\left(\left.\partial_{x_{n}} \varphi^{g}\right|_{x_{n}=0^{+}}\right)^{2}\right)\left(1+\frac{\left.\tilde{r}\left(x, \xi^{\prime}, \partial_{x^{\prime}} \varphi\right)^{2}\right|_{x_{n}=0^{+}}}{\left(\left.\partial_{x_{n}} \varphi^{d}\right|_{x_{n}=0^{+}}\right)^{2}\left(\left.\partial_{x_{n}} \varphi^{g}\right|_{x_{n}=0^{+}}\right)^{2}}\right) \\
& \geq C>0
\end{aligned}
$$

with the assumptions on $\varphi$ we just wrote. We can then use the same argument as in the proof of Proposition 2.7 and prove that the result of this proposition also holds in this case. The rest of the proof of Theorem 2.2 below remains unchanged. More generally, for other elliptic operators, the result of Theorem 2.2 holds if we can choose a weight function that yields the result of Proposition 2.7.

In particular, the Carleman estimate in Theorem 2.2 provides a quantitative result for the unique continuation property across the interface $S$ (See for instance [Hör63] or [Zui83]). 
2.3. Estimate in the region $\mathscr{E}^{d,+}$. With a microlocal cut-off, we place ourselves in the region region $\mathscr{E}^{d,+}$, hence in $\mathscr{E}^{g,+}$ by Proposition 2.7, and finitely away from $\mathscr{J}^{d}$ (and thus $\mathscr{J}^{g}$ ). Making use of the Calderónprojector technique we shall prove the following partial Carleman estimate.

Proposition 2.9. Let $K$ be a compact subset of $V$. Let $\chi^{+}\left(x_{0}, x, \xi_{0}, \xi^{\prime}\right) \in S_{\mathcal{T}}^{0}$ with a compact support w.r.t. $\left(x_{0}, x\right)$ contained in $V$, such that in the support of $\chi^{+}$we have $\mu^{d}\left(x_{0}, x, \xi_{0}, \xi^{\prime}\right) \geq C>0$. With the weight function $\varphi$ satisfying Assumption 2.1, there exist $C>0$ and $h_{1}>0$ such that

$$
\begin{aligned}
\left\|\mathrm{op}\left(\chi^{+}\right) v\right\|_{1}+\left.h^{\frac{1}{2}}\left|\mathrm{op}\left(\chi^{+}\right) v\right|_{x_{n}=0^{+}}\right|_{1}+\left.h^{\frac{1}{2}}\left|\operatorname{op}\left(\chi^{+}\right) D_{x_{n}} v\right|_{x_{n}=0^{+}}\right|_{0} \\
\leq C\left(\left\|P_{\varphi} v\right\|_{0}+h\|v\|_{1}+\left.h^{2}\left|D_{x_{n}} v\right|_{x_{n}=0^{+}}\right|_{0}+h^{\frac{1}{2}}\left|\theta_{\varphi}\right|_{1}+h^{\frac{1}{2}}\left|\Theta_{\varphi}\right|_{0}\right),
\end{aligned}
$$

for $0<h \leq h_{1}$, and for $v={ }^{t}\left(v^{g}, v^{d}\right), v^{g}, v^{d} \in \mathscr{C}_{c}^{\infty}\left(K^{d}\right)$ and satisfying $\left(\mathrm{TC}_{\varphi}\right)$.

The proof we give follows that of Lemma 4 in [LR97] and the notation used therein. We reproduce some of the arguments of [LR97] to have a self-contained proof of Proposition 2.9. Note that the first term in the partial estimate (2.19) differs from the equivalent term in the Carleman estimate (2.7) by a factor $h^{\frac{1}{2}}$. Here, a "better" estimate is actually obtained because we have restricted ourselves microlocally to an ellipticity region of the symbol $p_{\varphi}$. The Carleman estimate (2.7), for the second-order operator $A_{2}$, in fact corresponds to a sub-elliptic estimate.

Proof. In $\operatorname{supp}\left(\chi^{+}\right)$, we have

$$
\operatorname{Im} \rho^{g / d,+} \geq C>0, \quad \operatorname{Im} \rho^{g / d,-} \leq-C<0,
$$

by Lemma 2.5 and remark 2.6. Moreover, $\chi^{+} \rho^{g / d,+}$ and $\chi^{+} \rho^{g / d,-}$ are in $S_{\mathcal{T}}^{1}$.

We set $u=\mathrm{op}\left(\chi^{+}\right) v$. Then, $P_{\varphi} u=g$ with $g=\mathrm{op}\left(\chi^{+}\right) P_{\varphi} v+\underbrace{\left[P_{\varphi}, \mathrm{op}\left(\chi^{+}\right)\right]}_{\in h \Psi^{1}} v$. In particular, we have

$$
\|g\|_{0} \leq C\left(\left\|P_{\varphi} v\right\|_{0}+h\|v\|_{1}\right) .
$$

The transmission conditions satisfied by $u^{g}$ and $u^{d}$ are

$$
\left.u^{g}\right|_{x_{n}=0^{+}}=\left.u^{d}\right|_{x_{n}=0^{+}}+\theta_{\varphi, \chi^{+}},\left.\quad c^{g}\left(D_{x_{n}}+i \partial_{x_{n}} \varphi^{g}\right) u^{g}\right|_{x_{n}=0^{+}}+\left.c^{d}\left(D_{x_{n}}+i \partial_{x_{n}} \varphi^{d}\right) u^{d}\right|_{x_{n}=0^{+}}=G_{1},
$$

with $\theta_{\varphi, \chi^{+}}:=\left.\mathrm{op}\left(\chi^{+}\right) \theta_{\varphi}\right|_{x_{n}=0^{+}}$and

$$
G_{1}=\left.\underbrace{\left[c^{g}\left(D_{x_{n}}+i \partial_{x_{n}} \varphi^{g}\right), \mathrm{op}\left(\chi^{+}\right)\right]}_{\in h \Psi_{\mathcal{T}}^{0}} v^{g}\right|_{x_{n}=0^{+}}+\left.\underbrace{\left[c^{d}\left(D_{x_{n}}+i \partial_{x_{n}} \varphi^{d}\right), \mathrm{op}\left(\chi^{+}\right)\right]}_{\in h \Psi_{\mathcal{T}}^{0}} v^{d}\right|_{x_{n}=0^{+}}+\left.\mathrm{op}\left(\chi^{+}\right) \Theta_{\varphi}\right|_{x_{n}=0^{+}}
$$

that satisfies

$$
\left|G_{1}\right|_{0} \leq\left. C h|v|_{x_{n}=0^{+}}\right|_{0}+C\left|\Theta_{\varphi}\right|_{0} .
$$

We denote by $\underline{\phi}$ the zero-extension of a function $\phi \in \mathscr{C}^{\infty}\left(V^{d}\right)$ to $\mathbf{R}^{n+1}$. We then have

$$
P_{\varphi} \underline{u}=\underline{g}-h^{2} \gamma_{0}(u) \delta^{\prime}+\frac{h}{i}\left(\gamma_{1}(u)-\mathrm{op}\left(q_{\rho}\right) \gamma_{0}(u)\right) \delta, \quad \gamma_{0}(u):=\left.u\right|_{x_{n}=0^{+}}, \quad \gamma_{1}(u):=\left.D_{x_{n}} u\right|_{x_{n}=0^{+}},
$$


where $\delta^{(j)}=\left(\frac{d}{d x_{n}}\right)^{j} \delta_{x_{n}=0}$, and $q_{\rho}=\operatorname{diag}\left(\rho^{g,-}+\rho^{g,+}, \rho^{d,-}+\rho^{d,+}\right)$ since $\rho^{g / d,+}+\rho^{g / d,-}=-2 i \partial_{x_{n}} \varphi^{g / d}$. Setting

$$
w_{1}:=\gamma_{0}(u), \quad \text { and } \quad w_{0}=\gamma_{1}(u)-o p\left(q_{\rho}\right) \gamma_{0}(u)
$$

we write

$$
P_{\varphi} \underline{u}=\underline{g}-h^{2} w_{1} \delta^{\prime}+\frac{h}{i} w_{0} \delta .
$$

We now choose $\chi\left(x_{0}, x, \xi_{0}, \xi\right) \in S^{0}$ equal to one for sufficiently large $\left|\left(\xi_{0}, \xi\right)\right|$ as well as in a neighborhood of $\operatorname{supp}\left(\chi^{+}\right)$with moreover $\operatorname{supp}(\chi) \cap \operatorname{det}\left(p_{\varphi}\right)^{-1}(\{0\})=\emptyset$. These conditions are compatible from the choice made for $\operatorname{supp}\left(\chi^{+}\right)$and Proposition 2.7. From the ellipticity of $p_{\varphi}$ on $\operatorname{supp}(\chi)$, for large $M$, there exists a $\psi \mathrm{DO} E_{M}=\mathrm{Op}(e)$, with $e \in S^{-2}$, of the form $e=\sum_{j=0}^{M} h^{j} e_{j}$, with $e_{j} \in S^{-2-j}$ and $e_{0}^{g / d}=\chi / p_{\varphi}^{g / d}$, that satisfies

$$
E_{M} \circ P_{\varphi}=\mathrm{Op}(\chi)+h^{M+1} R_{M}, \quad R_{M} \in \Psi^{-1-M} .
$$

Note that the parametrix construction yields the symbols $e_{j}^{g / d}, j=0, \ldots, M$, in the form of rational functions for large $\left|\xi_{n}\right|$, with $\rho^{g / d,+}$ and $\rho^{g / d,-}$ for only poles.

With such a parametrix $E_{M}$ we obtain

$$
\underline{u}=E_{M} \underline{g}+E_{M}\left(-h^{2} w_{1} \delta^{\prime}+\frac{h}{i} w_{0} \delta\right)+g_{1}, \quad g_{1}=(\operatorname{Id}-\mathrm{Op}(\chi)) \underline{u}-h^{M+1} R_{M} \underline{u} .
$$

We have the following lemma.

Lemma 2.10. Let $\sigma\left(z, \zeta^{\prime}, h\right) \in S_{\mathcal{T}}^{m}$ and $\Sigma(z, \zeta, h) \in S^{-\infty} \operatorname{such}$ that $\operatorname{supp}(\sigma) \cap \operatorname{supp}(\Sigma)=\emptyset$. Then

$$
\operatorname{op}(\sigma) \circ \operatorname{Op}(\Sigma) \in \bigcap_{N \in \mathbf{N}} h^{N} \Psi^{-N}, \quad \text { and } \quad \mathrm{Op}(\Sigma) \circ \mathrm{op}(\sigma) \in \bigcap_{N \in \mathbf{N}} h^{N} \Psi^{-N} .
$$

Proof. We use the idea of the proof of Theorem 18.1.35 in [Hör85b]. From the remark preceding Theorem 18.1.17 in [Hör85b], adapted to semi-classical operators, we observe that

$$
\operatorname{op}(\sigma) \circ \mathrm{Op}(\Sigma) e^{i\langle z, \zeta\rangle / h}=\operatorname{op}(\sigma)\left(e^{i z_{n} \zeta_{n} / h} \Sigma\left(z, D_{z^{\prime}}, \zeta_{n}, h\right) e^{i\left\langle z^{\prime}, \zeta^{\prime}\right\rangle / h}\right)=e^{i z_{n} \zeta_{n} / h}\left(\operatorname{op}(\sigma) \circ \Sigma\left(z, D_{z^{\prime}}, \zeta_{n}, h\right)\right) e^{i\left\langle z^{\prime}, \zeta^{\prime}\right\rangle / h}
$$

where $\Sigma\left(z, D_{z^{\prime}}, \zeta_{n}, h\right)$ denotes the tangential operator $\operatorname{op}\left(\Sigma\left(z, \zeta, \zeta_{n}, h\right)\right)$ with $\zeta_{n}$ as a parameter. Since $\left\langle\zeta^{\prime}\right\rangle \leq$ $\langle\zeta\rangle$, we indeed observe that, for all $N \in \mathbf{N}, \Sigma\left(z, \zeta, \zeta_{n}, h\right)$ is bounded in $S_{\mathcal{T}}^{-N}$ uniformly w.r.t. $\zeta_{n} \in \mathbf{R}^{n}$. We set $\lambda\left(z, \zeta^{\prime}, \zeta_{n}, h\right):=\sigma \#_{\mathcal{T}} \Sigma$ as given in the composition formula (1.3), with $\zeta_{n}$ as a parameter. We hence have $\operatorname{op}(\sigma) \circ \mathrm{Op}(\Sigma) e^{i\langle z, \zeta\rangle / h}=e^{i\langle z, \zeta\rangle / h} \lambda(z, \zeta, h)$.

With $\operatorname{supp}(\sigma) \cap \operatorname{supp}(\Sigma)=\emptyset$, for all $M \in \mathbf{N}$, we find

$$
\begin{aligned}
\lambda(z, \zeta, h)=\frac{(-i h)^{M+1}}{(2 \pi h)^{n}} \sum_{|\alpha|=M+1} \int_{0}^{1} \frac{(M+1)(1-s)^{M}}{\alpha !} \iint e^{\left.-i\left\langle t^{\prime}, \tau^{\prime}\right\rangle\right) / h} \\
\quad \times \partial_{\zeta^{\prime}}^{\alpha} \sigma\left(z, \zeta^{\prime}+\tau^{\prime}, h\right) \partial_{z^{\prime}}^{\alpha} \Sigma\left(z^{\prime}+s t^{\prime}, z_{n}, \zeta^{\prime}, \zeta_{n}, h\right) d t^{\prime} d \tau^{\prime} d s .
\end{aligned}
$$

Note that for all $j \in \mathbf{N}, \partial_{z_{n}}^{j} \partial_{\zeta^{\prime}}^{\alpha} \sigma\left(z, \zeta^{\prime}, h\right)$ is in $S_{\mathcal{T}}^{m-|\alpha|}$, and that, for all $M^{\prime}, M^{\prime \prime}, j, k \in \mathbf{N},\left\langle\zeta_{n}\right\rangle^{M^{\prime}} \partial_{z_{n}}^{j} \partial_{\zeta_{n}}^{k} \partial_{z^{\prime}}^{\alpha} \Sigma(z, \zeta, h)$ is bounded in $S_{\mathcal{T}}^{-M^{\prime \prime}}$ uniformly w.r.t. $\zeta_{n}$ in $\mathbf{R}$. It follows that $\left\langle\zeta_{n}\right\rangle^{M^{\prime}} \partial_{z_{n}}^{j} \partial_{\zeta_{n}}^{k} \lambda(z, \zeta, h) \in h^{N} S_{\mathcal{T}}^{-N}$, for all $M^{\prime}, N, j, k \in$ 
N. Since $\langle\zeta\rangle \leq\left\langle\zeta^{\prime}\right\rangle\left\langle\zeta_{n}\right\rangle$, we see that $\lambda(z, \zeta, h)$ is a symbol in all variables and is in $\cap_{N \in \mathbf{N}} h^{N} S^{-N}$. We thus have $e^{i\langle z, \zeta\rangle / h} \lambda(z, \zeta, h)=\mathrm{Op}(\lambda) e^{i\langle z, \zeta\rangle / h}$ and therefore find

$$
\mathrm{op}(\sigma) \circ \mathrm{Op}(\Sigma) e^{i\langle z, \zeta\rangle / h}=\mathrm{Op}(\lambda) e^{i\langle z, \zeta\rangle / h}, \quad \text { for all } \zeta \in \mathbf{R}^{n+1} .
$$

Since both sides are continuous in $\mathscr{P}^{\prime}\left(\mathbf{R}^{n+1}\right)$ and linear combinations of exponential functions are dense in $\mathscr{P}^{\prime}$, we obtain op $(\sigma) \circ \mathrm{Op}(\Sigma)=\mathrm{Op}(\lambda)$.

To treat the other case, i.e., $\mathrm{Op}(\Sigma) \circ \mathrm{op}(\sigma)$, we prove that $\mathrm{op}(\sigma)^{*} \circ \mathrm{Op}(\Sigma)^{*} \in \cap_{N \in \mathbf{N}} h^{N} \Psi^{-N}$. We denote by $\sigma^{*}$ and $\Sigma^{*}$ the symbols of op $(\sigma)^{*}$ and $\mathrm{Op}(\Sigma)^{*}$. They are of the form

$$
\sigma^{*}=\tilde{\sigma}+\sigma_{\infty}, \quad \tilde{\sigma} \in S_{\mathcal{T}}^{m}, \quad \sigma_{\infty} \in \cap_{N \in \mathbf{N}} h^{N} S_{\mathcal{T}}^{-N}, \quad \text { and } \quad \Sigma^{*}=\tilde{\Sigma}+\Sigma_{\infty}, \quad \tilde{\Sigma} \in S^{-\infty}, \quad \Sigma_{\infty} \in \cap_{N \in \mathbf{N}} h^{N} S^{-N},
$$

where $\tilde{\sigma}$ and $\tilde{\Sigma}$ can be chosen such that $\operatorname{supp}(\tilde{\sigma}) \cap \operatorname{supp}(\tilde{\Sigma})=\emptyset$ from the $\psi \mathrm{DO}$ calculus. This yields

$$
\mathrm{op}(\sigma)^{*} \circ \mathrm{Op}(\Sigma)^{*}=\mathrm{op}(\tilde{\sigma}) \circ \mathrm{Op}(\tilde{\Sigma})+\mathrm{op}(\tilde{\sigma}) \circ \mathrm{Op}\left(\Sigma_{\infty}\right)+\mathrm{op}\left(\sigma_{\infty}\right) \circ \mathrm{Op}\left(\Sigma_{\infty}\right)+\mathrm{op}\left(\sigma_{\infty}\right) \circ \mathrm{Op}(\tilde{\Sigma}) .
$$

The first term is treated as above. The other terms can be treated similarly with formula (1.3) for $M=0$ : for the second and third terms we use that for all $M^{\prime}, M^{\prime \prime}, j, k \in \mathbf{N},\left\langle\zeta_{n}\right\rangle^{M^{\prime}} \partial_{z_{n}}^{j} \partial_{\zeta_{n}}^{k} \Sigma_{\infty}(z, \zeta, h)$ is bounded in $h^{M^{\prime \prime}} S_{\mathcal{T}}^{-M^{\prime \prime}}$ uniformly w.r.t. $\zeta_{n}$ in $\mathbf{R}$; for the fourth term we use that for all $j, N \in \mathbf{N}, \partial_{z_{n}}^{j} \sigma_{\infty}\left(z, \zeta^{\prime}, h\right)$ is in $h^{N} S_{\mathcal{T}}^{-N}$ and $\left\langle\zeta_{n}\right\rangle^{M^{\prime}} \partial_{z_{n}}^{j} \partial_{\zeta_{n}}^{k} \tilde{\Sigma}(z, \zeta, h)$ is bounded in $S_{\mathcal{T}}^{-N}$ uniformly w.r.t. $\zeta_{n}$ in $\mathbf{R}$, for all $M^{\prime}, N, j, k \in \mathbf{N}$.

Continuation of the proof of Proposition 2.9. With Lemma 2.10, we have $(\operatorname{Id}-\operatorname{Op}(\chi)) \circ \mathrm{op}\left(\chi^{+}\right) \in$ $\cap_{N \in \mathbf{N}} h^{N} \Psi^{-N}$. Noting that $\underline{u}=\mathrm{op}\left(\chi^{+}\right) \underline{v}$, we obtain

$$
\left\|g_{1}\right\|_{2} \leq C h^{2}\|v\|_{0} .
$$

Next, we compute the action in the region $x_{n}>0$ of the parametrix $E_{M}$ on the terms defined on the interface in (2.24). We find

$$
\begin{aligned}
& E_{M}\left(\frac{h}{i} w_{0} \delta\right)\left(x_{0}, x\right)=(2 \pi h)^{-n} \iint e^{i\left(\left(x_{0}-z_{0}\right) \xi_{0}+\left\langle x^{\prime}-z^{\prime}, \xi^{\prime}\right\rangle\right) / h} \hat{t}_{0}\left(x_{0}, x, \xi_{0}, \xi^{\prime}\right) w_{0}\left(z_{0}, z^{\prime}\right) d\left(z_{0}, z^{\prime}\right) d\left(\xi_{0}, \xi^{\prime}\right), \\
& E_{M}\left(-h^{2} w_{1} \delta^{\prime}\right)=(2 \pi h)^{-n} \iint e^{i\left(\left(x_{0}-z_{0}\right) \xi_{0}+\left\langle x^{\prime}-z^{\prime}, \xi^{\prime}\right\rangle\right) / h} \hat{t}_{1}\left(x_{0}, x, \xi_{0}, \xi^{\prime}\right) w_{1}\left(z_{0}, z^{\prime}\right) d\left(z_{0}, z^{\prime}\right) d\left(\xi_{0}, \xi^{\prime}\right),
\end{aligned}
$$

where

$$
\hat{t}_{0}\left(x_{0}, x, \xi_{0}, \xi^{\prime}\right)=\frac{1}{2 i \pi} \int_{\mathbf{R}} e^{i x_{n} \xi_{n} / h} e\left(x_{0}, x, \xi_{0}, \xi\right) d \xi_{n}, \quad \hat{t}_{1}\left(x_{0}, x, \xi_{0}, \xi^{\prime}\right)=\frac{1}{2 i \pi} \int_{\mathbf{R}} e^{i x_{n} \xi_{n} / h} e\left(x_{0}, x, \xi_{0}, \xi\right) \xi_{n} d \xi_{n}
$$

Note that the integral defining $\hat{t}_{0}$ is absolutely converging. The integral defining $\hat{t}_{1}$ is however to be understood in the sense of oscillatory integrals [Hör90, Section 7.8]. Note that we have

$$
\hat{t}_{1}\left(x_{0}, x, \xi_{0}, \xi^{\prime}\right)=\left.\frac{1}{2 i \pi} D_{z_{n}} \int_{\mathbf{R}} e^{i z_{n} \xi_{n} / h} e\left(x_{0}, x, \xi_{0}, \xi\right) d \xi_{n}\right|_{z_{n}=x_{n}} .
$$

The choice we have made for the cut-off function $\chi$ makes the symbol $e\left(x_{0}, x, \xi_{0}, \xi\right)$ holomorphic for large $\left|\xi_{n}\right|, \xi_{n} \in \mathbf{C}$. In $x_{n}>0$, we thus obtain

$$
\hat{t}_{0}\left(x_{0}, x, \xi_{0}, \xi^{\prime}\right)=\frac{1}{2 i \pi} \int_{\gamma} e^{i x_{n} \xi_{n} / h} e\left(x_{0}, x, \xi_{0}, \xi\right) d \xi_{n},
$$


where $\gamma$ is the union of the segment $\left\{\xi_{n} \in \mathbf{R} ;\left|\xi_{n}\right| \leq C_{0}\left|\left(\xi_{0}, \xi^{\prime}\right)\right|\right\}$ and the half circle $\left\{\xi_{n} \in \mathbf{C} ;\left|\xi_{n}\right|=\right.$ $\left.C_{0}\left|\left(\xi_{0}, \xi^{\prime}\right)\right|, \operatorname{Im} \xi_{n}>0\right\}$, where the constant $C_{0}$ is chosen sufficiently large so as to have the roots $\rho^{g /,+}+$ inside the domain with boundary $\gamma$ (recall that $\chi^{+} \rho^{g / d,+}$ is in $S^{1}$ ). From (2.26), we obtain similarly

$$
\hat{t}_{1}\left(x_{0}, x, \xi_{0}, \xi^{\prime}\right)=\frac{1}{2 i \pi} \int_{\gamma} e^{i x_{n} \xi_{n} / h} e\left(x_{0}, x, \xi_{0}, \xi\right) \xi_{n} d \xi_{n}, \quad x_{n}>0 .
$$

The expression (2.27) and (2.28) above are valid in $x_{n}>0$ but admit a trace at $x_{n}=0^{+}$. In particular, we note that we have

$$
\left|D_{x_{n}}^{l} \partial_{\left(x_{0}, x^{\prime}\right)}^{\alpha} \partial_{\left(\xi_{0}, \xi^{\prime}\right)}^{\beta} \hat{t}_{j}\right| \leq C_{\alpha, \beta, l}\left\langle\left(\xi_{0}, \xi^{\prime}\right)\right\rangle^{-1+j+l-|\beta|}, \quad x_{n} \geq 0, \quad j=0,1, \quad l \in \mathbf{N} .
$$

We now choose $\chi_{1}\left(x_{0}, x, \xi_{0}, \xi^{\prime}\right) \in S_{\mathcal{T}}^{0}$, satisfying the same requirement as $\chi^{+}$, equal to one in a neighborhood of $\operatorname{supp}\left(\chi^{+}\right)$and such that the symbol $\chi$ be equal to one in a neighborhood of $\operatorname{supp}\left(\chi_{1}\right)$. We set $t_{j}=\chi_{1} \hat{t}_{j}$, $j=0,1$ and $g_{2}=\mathrm{op}\left(\left(1-\chi_{1}\right) \hat{t}_{0}\right) w_{0}+\mathrm{op}\left(\left(1-\chi_{1}\right) \hat{t}_{1}\right) w_{1}$. This yields

$$
\underline{u}=E_{M} \underline{g}+\mathrm{op}\left(t_{0}\right) w_{0}+\mathrm{op}\left(t_{1}\right) w_{1}+g_{1}+g_{2} .
$$

From the composition formula of tangential operators (1.3), noting that it does not involve derivations w.r.t. the variable $x_{n}$, and estimate (2.29), we obtain

$$
\left\|g_{2}\right\|_{2} \leq C h^{2}\left(\|v\|_{1}+\left.\left|D_{x_{n}} v\right|_{x_{n}=0^{+}}\right|_{0}\right)
$$

since $\operatorname{supp}\left(1-\chi_{1}\right) \cap \operatorname{supp}\left(\chi^{+}\right)=\emptyset$, by making use of the following trace formula [LR97, page 486]

$$
\left.|\psi|_{x_{n}=0^{+}}\right|_{j} \leq C h^{-\frac{1}{2}}\|\psi\|_{j+1}, \quad j \in \mathbf{N} .
$$

We now observe that the symbols $e\left(x_{0}, x, \xi_{0}, \xi\right)$ is holomorphic w.r.t. $\xi_{n}$ in the support of $\chi_{1}$. We can then write

$$
t_{j}=\operatorname{diag}\left(t_{j}^{g}, t_{j}^{d}\right), \quad t_{j}^{g / d}\left(x_{0}, x, \xi_{0}, \xi^{\prime}\right)=\chi_{1}\left(x_{0}, x, \xi_{0}, \xi^{\prime}\right) \frac{1}{2 i \pi} \int_{\substack{\gamma_{/} \\ \gamma_{0}}} e^{i x_{n} \xi_{n} / h} e^{g / d}\left(x_{0}, x, \xi_{0}, \xi\right) \xi_{n}^{j} d \xi_{n}, \quad j=0,1,
$$

where $\gamma_{0}^{g / d}$ is a direct contour surrounding the roots $\rho^{g / d,+}$ in the region $\operatorname{Im} \xi_{n} \geq c_{0}\left|\left(\xi_{0}, \xi^{\prime}\right)\right|$, for $c_{0}>0$.

We note that in $\operatorname{supp}\left(\chi_{1}\right)$ we have

$$
e_{0}^{g / d}=\frac{1}{p_{\varphi}^{g / d}}=\frac{1}{\rho^{g / d,+}-\rho^{g / d,-}}\left(\frac{1}{\xi_{n}-\rho^{g / d,+}}-\frac{1}{\xi_{n}-\rho^{g / d,-}}\right) .
$$

The residue formula then yields

$$
e^{-i x_{n} \rho^{g / d,+} / h} t_{j}^{g / d}=\chi_{1} \frac{\left(\rho^{g / d,+}\right)^{j}}{\rho^{g / d,+}-\rho^{g / d,-}}+h \lambda^{g / d}, \quad j=0,1, \quad \lambda^{g / d} \in S_{\mathcal{T}}^{-2+j} .
$$

It should be noted that it is crucial to have $\operatorname{Im} \rho^{g / d,+} \geq C>0$ and $\operatorname{Im} \rho^{g / d,-} \leq-C<0$ here. From (2.33) we obtain the estimate

$$
\left|\left(D_{x_{n}}\right)^{l} \partial_{x_{0}, x^{\prime}}^{\alpha} \partial_{\xi_{0}, \xi^{\prime}}^{\beta} t_{j}\right| \leq C_{\alpha, \beta, l} e^{-c_{0}\left(x_{n} / h\right)\left\langle\left(\xi_{0}, \xi^{\prime}\right)\right\rangle}\left\langle\left(\xi_{0}, \xi^{\prime}\right)\right\rangle^{-1+j-|\beta|+l}, \quad x_{n} \geq 0,
$$


again by the residue formula, which yields $e^{c_{0} x_{n} / h}\left(D_{x_{n}}\right)^{l} t_{j}$ bounded in $S_{\mathcal{T}}^{j-1+l}$ uniformly w.r.t. $x_{n} \geq 0$. It follows that

$$
\left\|\Lambda^{1} \circ \mathrm{op}\left(t_{j}\right) w_{j}\right\|_{0}^{2}=\int_{x_{n}>0} e^{-2 c_{0} x_{n} / h}\left|\operatorname{op}\left(e^{c_{0} x_{n} / h} t_{j}\right) w_{j}\right|_{1}^{2}\left(x_{n}\right) d x_{n} \leq C h\left|w_{j}\right|_{j}^{2}
$$

and

$$
\left\|D_{x_{n}} \mathrm{op}\left(t_{j}\right) w_{j}\right\|_{0}^{2}=\int_{x_{n}>0} e^{-2 c_{0} x_{n} / h}\left|\operatorname{op}\left(e^{c_{0} x_{n} / h} D_{x_{n}} t_{j}\right) w_{j}\right|_{0}^{2}\left(x_{n}\right) d x_{n} \leq C h\left|w_{j}\right|_{j}^{2} .
$$

From (2.30), and estimates (2.20), (2.25), (2.31), (2.35), (2.36) we obtain

$$
\|u\|_{1} \leq C\left(\left\|P_{\varphi} v\right\|_{0}+h\|v\|_{1}+h^{\frac{1}{2}}\left(\left|w_{0}\right|_{0}+\left|w_{1}\right|_{1}\right)+\left.h^{2}\left|D_{x_{n}} v\right|_{x_{n}=0^{+}}\right|_{0}\right) .
$$

We shall now address the boundary terms $w_{0}$ and $w_{1}$. We take the trace at $x_{n}=0^{+}$of (2.30) which gives

$$
\gamma_{0}(u)=\mathrm{op}(a) \gamma_{0}(u)+\mathrm{op}(b) \gamma_{1}(u)+G_{2},
$$

where $a \in S_{\mathcal{T}}^{0}$ and $b \in S_{\mathcal{T}}^{-1}$, with principal symbols

$$
\begin{aligned}
& a_{0}=\operatorname{diag}\left(a_{0}^{g}, a_{0}^{d}\right), \quad \text { with } \quad a_{0}^{g / d}=-\left.\left(\chi_{1} \frac{\rho^{g / d,-}}{\rho^{g / d,+}-\rho^{g / d,-}}\right)\right|_{x_{n}=0^{+}}, \\
& b_{-1}=\operatorname{diag}\left(b_{-1}^{g}, b_{-1}^{d}\right), \quad \text { with } \quad b_{-1}^{g / d}=\left.\left(\chi_{1} \frac{1}{\rho^{g / d,}-\rho^{g / d},-}\right)\right|_{x_{n}=0^{+}},
\end{aligned}
$$

by (2.34) and (2.22). Note that the symbols $a$ and $b$ are diagonal. The function $G_{2}$ is given by $G_{2}=$ $\left.\left(E_{M} \underline{g}+g_{1}+g_{2}\right)\right|_{x_{n}=0^{+}}$. From the trace formula (2.32), we write

$$
\left|G_{2}\right|_{1} \leq C h^{-\frac{1}{2}}\left\|E_{M} \underline{g}+g_{1}+g_{2}\right\|_{2} \leq C h^{-\frac{1}{2}}\left(\left\|P_{\varphi} v\right\|_{0}+h\|v\|_{1}+\left.h^{2}\left|D_{x_{n}} v\right|_{x_{n}=0^{+}}\right|_{0}\right),
$$

since $E_{M} \in \Psi^{-2}$ and making use of estimates (2.20), (2.25) and (2.31).

The transmission conditions $\left(\mathrm{TC}_{u}\right)$ give

$$
\gamma_{0}\left(u^{g}\right)=\gamma_{0}\left(u^{d}\right)+\theta_{\varphi, \chi^{+}}, \quad \gamma_{1}\left(u^{g}\right)=-\beta \gamma_{1}\left(u^{d}\right)+k \gamma_{0}\left(u^{d}\right)+\tilde{G}_{1}
$$

where $\beta=\left.\left(c^{d} / c^{g}\right)\right|_{x_{n}=0^{+}}, k=-i\left(\left.\partial_{x_{n}} \varphi^{g}\right|_{x_{n}=0^{+}}+\left.\beta \partial_{x_{n}} \varphi^{d}\right|_{x_{n}=0^{+}}\right)$and $\tilde{G}_{1}=-i \partial_{x_{n}} \varphi^{g} \theta_{\varphi, \mathcal{\chi}^{+}}+\frac{1}{\left.c^{g}\right|_{x_{n}=0^{+}}} G_{1}$ with

$$
\left|\tilde{G}_{1}\right|_{0} \leq\left. C h|v|_{x_{n}=0^{+}}\right|_{0}+C\left(\left|\theta_{\varphi}\right|_{0}+\left|\Theta_{\varphi}\right|_{0}\right),
$$

by (2.21). From (2.38) we thus obtain

$$
(\operatorname{Id}-\mathrm{op}(a))\left(\begin{array}{c}
\gamma_{0}\left(u^{d}\right)+\theta_{\varphi, \chi^{+}} \\
\gamma_{0}\left(u^{d}\right)
\end{array}\right)=\mathrm{op}(b)\left(\begin{array}{c}
-\beta \gamma_{1}\left(u^{d}\right)+k \gamma_{0}\left(u^{d}\right)+\tilde{G}_{1} \\
\gamma_{1}\left(u^{d}\right)
\end{array}\right)+G_{2} .
$$

We thus have

$$
\left(\begin{array}{cc}
\mathrm{Id}-\mathrm{op}\left(a^{g}\right)-\mathrm{op}\left(b^{g}\right) \circ k & \mathrm{op}\left(b^{g}\right) \circ \beta \\
\mathrm{Id}-\mathrm{op}\left(a^{d}\right) & -\mathrm{op}\left(b^{d}\right)
\end{array}\right)\left(\begin{array}{l}
\gamma_{0}\left(u^{d}\right) \\
\gamma_{1}\left(u^{d}\right)
\end{array}\right)=G_{2}+\left(\begin{array}{c}
\mathrm{op}\left(a^{g}\right)-\mathrm{Id} \\
0
\end{array}\right) \theta_{\varphi, \chi^{+}}+\left(\begin{array}{c}
\mathrm{op}\left(b^{g}\right) \\
0
\end{array}\right) \tilde{G}_{1}
$$

where $\beta$ and $k$ stand here for the associated multiplication operators. We thus obtain a system of the form

$$
\operatorname{op}(\kappa)\left(\begin{array}{c}
\gamma_{0}\left(u^{d}\right) \\
\Lambda^{-1} \gamma_{1}\left(u^{d}\right)
\end{array}\right)=G_{2}+\mathrm{op}(\pi) \theta_{\varphi, \chi^{+}}+\operatorname{op}(\Pi) \tilde{G}_{1},
$$


where $\kappa$ is a $2 \times 2$ matrix with entries in $S_{\mathcal{T}}^{0}$, with principal symbol

$$
\kappa_{0}=\left(\begin{array}{cc}
1-a_{0}^{g}-k b_{-1}^{g} & \beta\left\langle\left(\xi_{0}, \xi^{\prime}\right)\right\rangle b_{-1}^{g} \\
1-a_{0}^{d} & -\left\langle\left(\xi_{0}, \xi^{\prime}\right)\right\rangle b_{-1}^{d}
\end{array}\right),
$$

and $\pi$ and $\Pi$ are $2 \times 1$ matrices with entries in $S_{\mathcal{T}}^{0}$ and $S_{\mathcal{T}}^{-1}$ respectively, with principal symbols

$$
\pi_{0}=\left(\begin{array}{c}
a_{0}^{g}-1 \\
0
\end{array}\right) \quad \text { and } \quad \Pi_{-1}=\left(\begin{array}{c}
b_{-1}^{g} \\
0
\end{array}\right) .
$$

We now choose $\chi_{2}\left(x_{0}, x, \xi_{0}, \xi^{\prime}\right) \in S_{\mathcal{T}}^{0}$, satisfying the same requirement as $\chi^{+}$, equal to one in a neighborhood of $\operatorname{supp}\left(\chi^{+}\right)$and such that the symbol $\chi_{1}$ be equal to one in a neighborhood of $\operatorname{supp}\left(\chi_{2}\right)$. In $\operatorname{supp}\left(\chi_{2}\right)$, we obtain

$$
\kappa_{0}\left|\operatorname{supp}\left(\chi_{2}\right)=\left(\begin{array}{ll}
\frac{\rho^{g,+}-k}{\rho^{g,+}-\rho^{g,-}} & \beta\left\langle\left(\xi_{0}, \xi^{\prime}\right)\right\rangle \frac{1}{\rho^{g,+}-\rho^{g,-}} \\
\frac{\rho^{d,+}}{\rho^{d,+}-\rho^{d,-}} & -\left\langle\left(\xi_{0}, \xi^{\prime}\right)\right\rangle \frac{1}{\rho^{d,+}-\rho^{d,-}}
\end{array}\right)\right|_{x_{n}=0^{+}} .
$$

This yields

$$
\left.\operatorname{det}\left(\kappa_{0}\right)\right|_{\operatorname{supp}\left(\chi_{2}\right)}=-\left.\frac{\left\langle\left(\xi_{0}, \xi^{\prime}\right)\right\rangle\left(\rho^{g,+}+\beta \rho^{d,+}-k\right)}{\left(\rho^{g,+}-\rho^{g,-}\right)\left(\rho^{d,+}-\rho^{d,-}\right)}\right|_{x_{n}=0^{+}} .
$$

Since we have $\operatorname{Im}\left(\rho^{g,+}+\beta \rho^{d,+}\right) \geq C\left\langle\left(\xi_{0}, \xi^{\prime}\right)\right\rangle>0$ in $\operatorname{supp}\left(\chi_{2}\right)$, and since

$$
\operatorname{Im}(-k)=\left.\frac{1}{\left.c^{g}\right|_{x_{n}=0^{+}}}\left(c^{g} \partial_{x_{n}} \varphi^{g}+c^{d} \partial_{x_{n}} \varphi^{d}\right)\right|_{x_{n}=0^{+}} \geq 0,
$$

by (2.10), we find that $\left|\operatorname{det}\left(\kappa_{0}\right)\right|_{\operatorname{supp}\left(\chi_{2}\right)} \mid \geq C>0$. It follows that $\kappa$ is elliptic in $\operatorname{supp}\left(\chi_{2}\right)$. Then, there exists $l_{M} \in S_{\mathcal{T}}^{0}$, such that

$$
\mathrm{op}\left(l_{M}\right) \circ \mathrm{op}(\kappa)=\mathrm{op}\left(\chi_{2}\right)+h^{M+1} \tilde{R}_{M}
$$

with $\tilde{R}_{M} \in \Psi_{\mathcal{T}}^{-1-M}$, for $M \in \mathbf{N}$ large. This yields

$$
\left(\begin{array}{c}
\gamma_{0}\left(u^{d}\right) \\
\Lambda^{-1} \gamma_{1}\left(u^{d}\right)
\end{array}\right)=\operatorname{op}\left(l_{M}\right) G_{2}+\mathrm{op}\left(l_{M}\right) \circ \mathrm{op}(\pi) \theta_{\varphi, \chi^{+}}+\mathrm{op}\left(l_{M}\right) \circ \mathrm{op}(\Pi) \tilde{G}_{1}+\left(\operatorname{op}\left(1-\chi_{2}\right)-h^{M} \tilde{R}_{M}\right)\left(\begin{array}{c}
\gamma_{0}\left(u^{d}\right) \\
\Lambda^{-1} \gamma_{1}\left(u^{d}\right)
\end{array}\right) \text {. }
$$

From the $\psi \mathrm{DO}$ calculus, since $\operatorname{supp}\left(1-\chi_{2}\right) \cap \operatorname{supp}\left(\chi^{+}\right)=\emptyset$, and making use of the trace formula (2.32) we obtain

$$
\begin{aligned}
\left|\gamma_{0}\left(u^{d}\right)\right|_{1}+\left|\gamma_{1}\left(u^{d}\right)\right|_{0} & \leq C\left(\left|G_{2}\right|_{1}+\left|\theta_{\varphi, \chi^{+}}\right|_{1}+\left|\tilde{G}_{1}\right|_{0}+h^{2}\|v\|_{1}+\left.h^{2}\left|D_{x_{n}} v\right|_{x_{n}=0^{+}}\right|_{0}\right) \\
& \leq C^{\prime}\left(h^{-\frac{1}{2}}\left\|P_{\varphi} v\right\|_{0}+h^{\frac{1}{2}}\|v\|_{1}+\left.h^{\frac{3}{2}}\left|D_{x_{n}} v\right|_{x_{n}=0^{+}}\right|_{0}+\left|\theta_{\varphi}\right|_{1}+\left|\Theta_{\varphi}\right|_{0}\right),
\end{aligned}
$$

by (2.41) and (2.39). From (2.40), the same estimate holds for $\left|\gamma_{0}\left(u^{g}\right)\right|_{1}+\left|\gamma_{1}\left(u^{g}\right)\right|_{0}$, and also for $\left|w_{0}\right|_{0}+\left|w_{1}\right|_{1}$ by (2.22):

$$
h^{\frac{1}{2}}\left(\left|w_{0}\right|_{0}+\left|w_{1}\right|_{1}\right) \leq C\left(\left\|P_{\varphi} v\right\|_{0}+h\|v\|_{1}+\left.h^{2}\left|D_{x_{n}} v\right|_{x_{n}=0^{+}}\right|_{0}+h^{\frac{1}{2}}\left|\theta_{\varphi}\right|_{1}+h^{\frac{1}{2}}\left|\Theta_{\varphi}\right|_{0}\right) .
$$

Observing that

$$
D_{x_{n}} \mathrm{op}\left(\chi^{+} v\right)=\mathrm{op}\left(\chi^{+}\right) D_{x_{n}} v+\underbrace{\left[D_{x_{n}}, \mathrm{op}\left(\chi^{+}\right)\right]}_{\in h \Psi_{\mathcal{T}}^{0}} v
$$


we have

$$
\begin{aligned}
h^{\frac{1}{2}}\left(\left.\left|\mathrm{op}\left(\chi^{+}\right) v\right|_{x_{n}=0^{+}}\right|_{1}\right. & \left.+\left.\left|\mathrm{op}\left(\chi^{+}\right) D_{x_{n}} v\right|_{x_{n}=0^{+}}\right|_{0}\right) \leq C h^{\frac{1}{2}}\left(\left.|u|_{x_{n}=0^{+}}\right|_{1}+\left.\left|D_{x_{n}} u\right|_{x_{n}=0^{+}}\right|_{0}+\left.h|v|_{x_{n}=0^{+}}\right|_{0}\right) \\
& \leq C\left(\left\|P_{\varphi} v\right\|_{0}+h\|v\|_{1}+\left.h^{2}\left|D_{x_{n}} v\right|_{x_{n}=0^{+}}\right|_{0}+h^{\frac{1}{2}}\left|\theta_{\varphi}\right|_{1}+h^{\frac{1}{2}}\left|\Theta_{\varphi}\right|_{0}\right),
\end{aligned}
$$

from the previous inequalities and the trace formula (2.32). We conclude the proof by combining estimates (2.37), (2.44) and (2.45).

2.4. Estimate in the region $\mathscr{E}^{d,-}$. With a microlocal cut-off, we place ourselves in the region $\mathscr{E}^{d,-}$, finitely away from $\mathcal{g}^{d}$. Making use of the standard techniques to prove Carleman estimates for both $P_{\varphi}^{g}$ and $P_{\varphi}^{d}$, we obtain the following partial Carleman estimate.

Proposition 2.11. Let $K$ be a compact subset of $V$. Let $\chi^{-}\left(x_{0}, x, \xi_{0}, \xi^{\prime}\right) \in S_{\mathcal{T}}^{0}$ with a compact support w.r.t. $\left(x_{0}, x\right)$ contained in $V$, be such that in the support of $\chi^{-}$we have $\mu^{d}\left(x_{0}, x, \xi_{0}, \xi^{\prime}\right) \leq-C<0$. With the weight function $\varphi$ satisfying Assumption 2.1, there exist $C>0$ and $h_{1}>0$ such that

$$
h\left\|\mathrm{op}\left(\chi^{-}\right) v\right\|_{1}^{2}+\left.h\left|\mathrm{op}\left(\chi^{-}\right) v\right|_{x_{n}=0^{+}}\right|_{1} ^{2}+\left.h\left|\mathrm{op}\left(\chi^{-}\right) D_{x_{n}} v\right|_{x_{n}=0^{+}}\right|_{0} ^{2} \leq C\left(\left\|P_{\varphi} v\right\|_{0}^{2}+h^{2}\|v\|_{1}^{2}+h\left|\theta_{\varphi}\right|_{1}^{2}+h\left|\Theta_{\varphi}\right|_{0}^{2}\right),
$$
for $0<h \leq h_{1}$, and for $v={ }^{t}\left(v^{g}, v^{d}\right), v^{d}, v^{g} \in \mathscr{C}_{c}^{\infty}\left(K^{d}\right)$ and satisfying $\left(\mathrm{TC}_{\varphi}\right)$.

Proof. We set $u=\mathrm{op}\left(\chi^{-}\right) v$. Then, $P_{\varphi} u=g$ with $g=\mathrm{op}\left(\chi^{-}\right) P_{\varphi} v+\left[P_{\varphi}, \mathrm{op}\left(\chi^{-}\right)\right] v$. In particular, we have

$$
\|g\|_{0} \leq C\left(\left\|P_{\varphi} v\right\|_{0}+h\|v\|_{1}\right) .
$$

The transmission conditions satisfied by $u^{d}$ and $u^{g}$ are $\left(\mathrm{TC}_{u}\right)$-see the proof of Proposition 2.9- with $\theta_{\varphi, \chi^{-}}:=$ op $\left.\left(\chi^{-}\right) \theta_{\varphi}\right|_{x_{n}=0^{+}}$in place of $\theta_{\varphi, \chi^{+}}$and with $G_{1}$ here given by

$$
G_{1}=\left.\left[c^{g}\left(D_{x_{n}}+i \partial_{x_{n}} \varphi^{g}\right), \mathrm{op}\left(\chi^{-}\right)\right] v^{g}\right|_{x_{n}=0^{+}}+\left.\left[c^{d}\left(D_{x_{n}}+i \partial_{x_{n}} \varphi^{d}\right), \operatorname{op}\left(\chi^{-}\right)\right] v^{d}\right|_{x_{n}=0^{+}}+\left.\operatorname{op}\left(\chi^{-}\right) \Theta_{\varphi}\right|_{x_{n}=0^{+}},
$$

and satisfying

$$
\left|G_{1}\right|_{0} \leq\left. C h|v|_{x_{n}=0^{+}}\right|_{0}+C\left|\Theta_{\varphi}\right|_{0} .
$$

We apply the Carleman method to the operators $P_{\varphi}^{g}$ and $P_{\varphi}^{d}$. By Assumption 2.1, and in particular by (2.3), and by Lemma 2 in [LR95], we then have

$$
\left.h\left\|u^{g / d}\right\|_{1}^{2}+\operatorname{Re}\left(h \mathscr{B}^{g / d}\left(u^{g / d}\right)+h^{2}\left(\left.\left(D_{n} u^{g / d}+L_{1}^{g / d} u^{g / d}\right)\right|_{x_{n}=0^{+}},\left.L_{0}^{g / d} u^{g / d}\right|_{x_{n}=0^{+}}\right)\right)_{0}\right) \leq C\left\|g^{g / d}\right\|_{0}^{2},
$$

for $h$ sufficiently small, where $L_{1}^{g / d} \in \mathscr{D}_{\mathcal{T}}^{1}, L_{0}^{g / d} \in \Psi_{\mathcal{T}}^{0}$. The quadratic forms $\mathscr{B}^{g / d}$ are given by

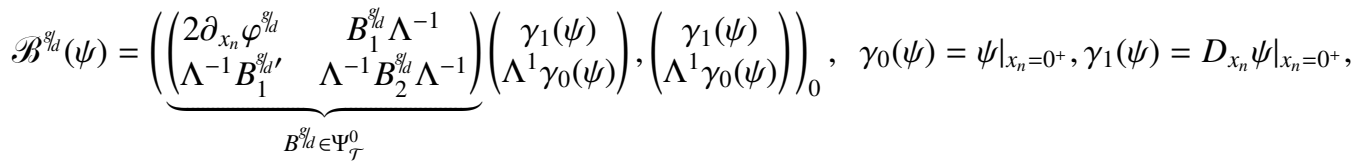

where $B_{1}^{g / d}, B_{1}^{g / d^{\prime}} \in \mathscr{D}_{\mathcal{T}}^{1}$, with $\sigma\left(B_{1}^{g / d}\right)=\sigma\left(B_{1}^{g / d^{\prime}}\right)=2 q_{1}^{g / d}$ and $B_{2}^{g / d} \in \mathscr{D}_{\mathcal{T}}^{2}$, with $\sigma\left(B_{2}^{g / d}\right)=-2 \partial_{x_{n}} \varphi^{g / d} q_{2}^{g / d}$. Observe that we have

$$
h^{2}\left|\left(\left.\left(D_{n} u^{g / d}+L_{1}^{g / d} u^{g / d}\right)\right|_{x_{n}=0^{+}},\left.L_{0}^{g / d} u^{g / d}\right|_{x_{n}=0^{+}}\right)\right| \leq C h^{2}\left(\left|\gamma_{1}\left(u^{g / d}\right)\right|_{0}^{2}+\left|\gamma_{0}\left(u^{g / d}\right)\right|_{1}^{2}\right) .
$$


The tangential $\psi$ DOs $B^{g / d}$ defined in (2.50) are of order 0 and their principal symbols are

$$
\sigma\left(B^{g / d}\right)=\left(\begin{array}{cc}
2 \partial_{x_{n}} \varphi^{g / d} & 2 q_{1}^{g / d}\left\langle\left(\xi_{0}, \xi^{\prime}\right)\right\rangle^{-1} \\
2 q_{1}^{g / d}\left\langle\left(\xi_{0}, \xi^{\prime}\right)\right\rangle^{-1} & -2 \partial_{x_{n}} \varphi^{g / d} q_{2}^{g / d}\left\langle\left(\xi_{0}, \xi^{\prime}\right)\right\rangle^{-2}
\end{array}\right) .
$$

We find $\operatorname{det}\left(\sigma\left(B^{g / d}\right)\right)=-4\left(\partial_{x_{n}} \varphi^{g / d}\right)^{2}\left\langle\left(\xi_{0}, \xi^{\prime}\right)\right\rangle^{-2} \mu^{g / d}$, with $\mu^{g / d}$ as defined in Section 2.2; it follows that in $\operatorname{supp}\left(\chi^{-}\right)$we have $\operatorname{det}\left(\sigma\left(B^{d}\right)\right) \geq C>0$. Since $\partial_{x_{n}} \varphi^{d}>0$ it follows that $\sigma\left(B^{d}\right)$ is positive definite.

We now make use of transmission conditions $\left(\mathrm{TC}_{u}\right)$ and write

$$
\left(\begin{array}{c}
\gamma_{1}\left(u^{g}\right) \\
\Lambda^{1} \gamma_{0}\left(u^{g}\right)
\end{array}\right)=\underbrace{\left(\begin{array}{cc}
-\beta & k \Lambda^{-1} \\
0 & 1
\end{array}\right)}_{C^{g}}\left(\begin{array}{c}
\gamma_{1}\left(u^{d}\right) \\
\Lambda^{1} \gamma_{0}\left(u^{d}\right)
\end{array}\right)+\left(\begin{array}{c}
\tilde{G}_{1} \\
\Lambda^{1} \theta_{\varphi, \mathcal{X}^{-}}
\end{array}\right)
$$

where $\beta=\left.\left(c^{d} / c^{g}\right)\right|_{x_{n}=0^{+}}, k=-i\left(\left.\partial_{x_{n}} \varphi^{g}\right|_{x_{n}=0^{+}}+\left.\beta \partial_{x_{n}} \varphi^{d}\right|_{x_{n}=0^{+}}\right)$and $\tilde{G}_{1}=-i \partial_{x_{n}} \varphi^{g} \theta_{\varphi, \chi^{-}}+\frac{1}{\left.c^{g}\right|_{x_{n}=0^{+}}} G_{1}$ that satisfies

$$
\left|\tilde{G}_{1}\right|_{0} \leq C h^{\frac{1}{2}}\|v\|_{1}+C\left(\left|\theta_{\varphi}\right|_{0}+\left|\Theta_{\varphi}\right|_{0}\right),
$$

by (2.48) and trace formula (2.32). We obtain

$$
\left.\mathscr{B}^{g}\left(u^{g}\right)=\left(\tilde{B}^{g}\left(\begin{array}{c}
\gamma_{1}\left(u^{d}\right) \\
\Lambda^{1} \gamma_{0}\left(u^{d}\right)
\end{array}\right),\left(\begin{array}{c}
\gamma_{1}\left(u^{d}\right) \\
\Lambda^{1} \gamma_{0}\left(u^{d}\right)
\end{array}\right)\right)\right)_{0}+U\left(\gamma_{1}\left(u^{d}\right), \gamma_{0}\left(u^{d}\right), \theta_{\varphi, \chi^{-}}, \tilde{G}_{1}\right)
$$

where $\sigma\left(\tilde{B}^{g}\right)=t \overline{\sigma\left(C^{g}\right)} \sigma\left(B^{g}\right) \sigma\left(C^{g}\right) \in S_{\mathcal{T}}^{1}$, which gives

$$
\left|\left(\tilde{B}^{g}\left(\begin{array}{c}
\gamma_{1}\left(u^{d}\right) \\
\Lambda^{1} \gamma_{0}\left(u^{d}\right)
\end{array}\right),\left(\begin{array}{c}
\gamma_{1}\left(u^{d}\right) \\
\Lambda^{1} \gamma_{0}\left(u^{d}\right)
\end{array}\right)\right)_{0}\right| \leq C\left(\left|\gamma_{0}\left(u^{d}\right)\right|_{1}^{2}+\left|\gamma_{1}\left(u^{d}\right)\right|_{0}^{2}\right),
$$

and where we have

$$
\begin{aligned}
U\left(\gamma_{1}\left(u^{d}\right), \gamma_{0}\left(u^{d}\right), \theta_{\varphi, \chi^{-}}, \tilde{G}_{1}\right) & =\left(B^{g} C^{g}\left(\begin{array}{c}
\gamma_{1}\left(u^{d}\right) \\
\Lambda^{1} \gamma_{0}\left(u^{d}\right)
\end{array}\right),\left(\begin{array}{c}
\tilde{G}_{1} \\
\Lambda^{1} \theta_{\varphi, \chi^{-}}
\end{array}\right)\right)_{0}+\left(B^{g}\left(\begin{array}{c}
\tilde{G}_{1} \\
\Lambda^{1} \theta_{\varphi, \chi^{-}}
\end{array}\right), C^{g}\left(\begin{array}{c}
\gamma_{1}\left(u^{d}\right) \\
\Lambda^{1} \gamma_{0}\left(u^{d}\right)
\end{array}\right)\right)_{0} \\
& +\left(B^{g}\left(\begin{array}{c}
\tilde{G}_{1} \\
\Lambda^{1} \theta_{\varphi, \chi^{-}}
\end{array}\right),\left(\begin{array}{c}
\tilde{G}_{1} \\
\Lambda^{1} \theta_{\varphi, \chi^{-}}
\end{array}\right)\right)_{0},
\end{aligned}
$$

which from (2.53) satisfies

$$
\left|U\left(\gamma_{1}\left(u^{d}\right), \gamma_{0}\left(u^{d}\right), \theta_{\varphi, \chi^{-}}, \tilde{G}_{1}\right)\right| \leq C\left(\left|\gamma_{0}\left(u^{d}\right)\right|_{1}^{2}+\left|\gamma_{1}\left(u^{d}\right)\right|_{0}^{2}+h\|v\|_{1}^{2}+\left|\theta_{\varphi}\right|_{1}^{2}+\left|\Theta_{\varphi}\right|_{0}^{2}\right) .
$$

For any $K>0$, with $\alpha>0$ sufficiently large, we can enforce $\alpha \sigma\left(B^{d}\right)+\sigma\left(\tilde{B}^{g}\right) \geq K>0$. Hence, with (2.54), (2.55) and Gårding's inequality [Tay81, Mar02] we obtain

$$
\alpha \operatorname{Re} \mathscr{B}^{d}\left(u^{d}\right)+\operatorname{Re} \mathscr{B}^{g}\left(u^{g}\right) \geq \frac{K}{2}\left(\left|\gamma_{0}\left(u^{d}\right)\right|_{1}^{2}+\left|\gamma_{1}\left(u^{d}\right)\right|_{0}^{2}\right)-\left(h\|v\|_{1}^{2}+\left|\theta_{\varphi}\right|_{1}^{2}+\left|\Theta_{\varphi}\right|_{0}^{2}\right),
$$

for $K$ sufficiently large and for $h$ sufficiently small. The transmission conditions (2.52) give

$$
\left|\gamma_{0}\left(u^{g}\right)\right|_{1}^{2}+\left|\gamma_{1}\left(u^{g}\right)\right|_{0}^{2} \leq C\left(\left|\gamma_{0}\left(u^{d}\right)\right|_{1}^{2}+\left|\gamma_{1}\left(u^{d}\right)\right|_{0}^{2}+\left|\theta_{\varphi}\right|_{1}^{2}+\left|\Theta_{\varphi}\right|_{0}^{2}+h\|v\|_{1}^{2}\right) \text {. }
$$

Recalling that

$$
D_{x_{n}} \operatorname{op}\left(\chi^{-} v\right)=\operatorname{op}\left(\chi^{-}\right) D_{x_{n}} v+\underbrace{\left[D_{x_{n}}, \mathrm{op}\left(\chi^{-}\right)\right]}_{\in h \Psi_{\mathcal{T}}^{0}} v,
$$

with the linear combination $\alpha(2.49)^{d}+(2.49)^{g}$ and estimates (2.47), (2.51), (2.56) and (2.57) we obtain the sought partial Carleman estimate, by choosing $\varepsilon$ and $h$ sufficiently small. 
Remark 2.12. As an alternative proof of Proposition 2.11, we could also use the Calderón projector technique for $P_{\varphi}^{d}$. In $\operatorname{supp}\left(\chi^{-}\right)$the two roots $\rho^{d, \pm}$ of $p_{\varphi}^{d}$ have negative imaginary part. With the notation and the argumentation of the proof of Proposition 2.9 above, the operators $t_{0}^{d}$ and $t_{1}^{d}$ vanish in $x_{n}>0$. The counterpart of (2.30) is then

$$
u^{d}=E_{M} \underline{g}^{d}+g_{1}^{d}+g_{2}^{d}, \quad \text { for } x_{n}>0 .
$$

We then obtain (see (2.37))

$$
\left\|u^{d}\right\|_{1} \leq C\left(\left\|P_{\varphi}^{d} v^{d}\right\|_{0}+h\left\|v^{d}\right\|_{1}+\left.h^{2}\left|D_{x_{n}} v^{d}\right|_{x_{n}=0^{+}}\right|_{0}\right) .
$$

We take the trace at $x_{n}=0^{+}$of $(2.58)$,

$$
\gamma_{0}\left(u^{d}\right)=G_{2}^{d}=\left.\left(E_{M} \underline{g}^{d}+g_{1}^{d}+g_{2}^{d}\right)\right|_{x_{n}=0^{+}},
$$

which, by the counterpart of (2.39), gives

$$
h^{\frac{1}{2}}\left|\gamma_{0}\left(u^{d}\right)\right|_{1} \leq C\left(\left\|P_{\varphi} v\right\|_{0}+h\|v\|_{1}+\left.h^{2}\left|D_{x_{n}} v\right|_{x_{n}=0^{+}}\right|_{0}\right) .
$$

From (2.58) we also have

$$
D_{x_{n}} u^{d}=D_{x_{n}} E_{M} \underline{g}^{d}+D_{x_{n}} g_{1}^{d}+D_{x_{n}} g_{2}^{d}, \quad \text { for } x_{n}>0 .
$$

We take the trace at $x_{n}=0^{+}$and obtain

$$
\gamma_{1}\left(u^{d}\right)=\left.\left(D_{x_{n}}\left(E_{M} \underline{g}^{d}+g_{1}^{d}+g_{2}^{d}\right)\right)\right|_{x_{n}=0^{+}} .
$$

From the trace formula (2.32) we then have

$$
\left|\gamma_{1}\left(u^{d}\right)\right|_{0} \leq C h^{-\frac{1}{2}}\left\|D_{x_{n}}\left(E_{M} \underline{g}^{d}+g_{1}^{d}+g_{2}^{d}\right)\right\|_{1} \leq C h^{-\frac{1}{2}}\left\|E_{M} \underline{g}^{d}+g_{1}^{d}+g_{2}^{d}\right\|_{2}
$$

and, by the counterpart of (2.39), this yields

$$
h^{\frac{1}{2}}\left|\gamma_{1}\left(u^{d}\right)\right|_{0} \leq C\left(\left\|P_{\varphi} v\right\|_{0}+h\|v\|_{1}+\left.h^{2}\left|D_{x_{n}} v\right|_{x_{n}=0^{+}}\right|_{0}\right) .
$$

From (2.60) and (2.61) and transmission condition $\left(\mathrm{TC}_{u}\right)$, we obtain

$$
h^{\frac{1}{2}}\left|\gamma_{0}\left(u^{g}\right)\right|_{1}+h^{\frac{1}{2}}\left|\gamma_{1}\left(u^{g}\right)\right|_{0} \leq C\left(\left\|P_{\varphi} v\right\|_{0}+h\|v\|_{1}+\left.h^{2}\left|D_{x_{n}} v\right|_{x_{n}=0^{+}}\right|_{0}+h^{\frac{1}{2}}\left|\theta_{\varphi, \chi^{-}}\right|_{1}+h^{\frac{1}{2}}\left|\Theta_{\varphi}\right|_{0}\right) .
$$

Finally with (2.59), (2.62), (2.51) , and (2.49) ${ }^{g}$ we can also achieve the result of Proposition 2.11.

2.5. Estimate around the region $\mathscr{Z}^{d}$. With a microlocal cut-off, we place ourselves in a neighborhood of the region $\mathscr{J}^{d}$, i.e., where $\left|\mu^{d}\right|$ is small, while staying in the region $\mathscr{E}^{g,+}$ away from the region $\mathscr{J}^{g}$. Making use of the techniques of Calderón projectors for $P_{\varphi}^{g}$ and standard techniques to prove Carleman estimates for $P_{\varphi}^{d}$, we obtain the following partial Carleman estimate.

Proposition 2.13. Let $K$ be a compact subset of $V$. Let $\varphi$ satisfy Assumption 2.1. Let $\chi^{0}\left(x_{0}, x, \xi_{0}, \xi^{\prime}\right) \in$ $S_{\mathcal{T}}^{0}$ with a compact support w.r.t. $\left(x_{0}, x\right)$ contained in $V$ and be such that in the support of $\chi^{0}$ we have $\mu^{g}\left(x_{0}, x, \xi_{0}, \xi^{\prime}\right) \geq C>0$ and

$$
\left.\left(c^{g} \partial_{x_{n}} \varphi^{g}+c^{d} \partial_{x_{n}} \varphi^{d}\right)^{2}\right|_{x_{n}=0^{+}}-\left.\left(c^{d}\right)^{2} \mu^{d}\right|_{x_{n}=0^{+}} \geq C^{\prime}>0, \quad \text { in } \operatorname{supp}\left(\chi^{0}\right) .
$$


Then, there exist $C>0$ and $h_{1}>0$ such that

(2.64) $h\left\|\mathrm{op}\left(\chi^{0}\right) v\right\|_{1}^{2}+\left.h\left|\mathrm{op}\left(\chi^{0}\right) v\right|_{x_{n}=0^{+}}\right|_{1} ^{2}+\left.h\left|\mathrm{op}\left(\chi^{0}\right) D_{x_{n}} v\right|_{x_{n}=0^{+}}\right|_{0} ^{2}$

$$
\leq C\left(\left\|P_{\varphi} v\right\|_{0}^{2}+h^{2}\|v\|_{1}^{2}+\left.h^{4}\left|D_{x_{n}} v\right|_{x_{n}=0^{+}}\right|_{0} ^{2}+h\left|\theta_{\varphi}\right|_{1}^{2}+h\left|\Theta_{\varphi}\right|_{0}^{2}\right),
$$

for $0<h \leq h_{1}$, and for $v={ }^{t}\left(v^{g}, v^{d}\right), v^{d}, v^{g} \in \mathscr{C}_{c}^{\infty}\left(K^{d}\right)$ and satisfying $\left(\mathrm{TC}_{\varphi}\right)$.

Proof. Condition (2.63) can be obtained from the properties of the weight function $\varphi$ listed in Assumption 2.1. In $\operatorname{supp}\left(\chi^{0}\right)$, we have

$$
\operatorname{Im} \rho^{g,+} \geq C>0, \quad \operatorname{Im} \rho^{g,-} \leq-C<0 .
$$

We set $u=\mathrm{op}\left(\chi^{0}\right) v$. Then, $P_{\varphi} u=g$ with $g=\mathrm{op}\left(\chi^{0}\right) P_{\varphi} v+\left[P_{\varphi}, \mathrm{op}\left(\chi^{0}\right)\right] v$. In particular, we have

$$
\left\|g^{g / d}\right\|_{0} \leq C\left(\left\|P_{\varphi}^{g / d} v^{g / d}\right\|_{0}+h\left\|v^{g / d}\right\|_{1}\right) .
$$

The transmission conditions satisfied by $u^{d}$ and $u^{g}$ are $\left(\mathrm{TC}_{u}\right)$-see the proof of Proposition 2.9- with $\theta_{\varphi, \chi^{0}}:=$ $\left.\mathrm{op}\left(\chi^{0}\right) \theta_{\varphi}\right|_{x_{n}=0^{+}}$in place of $\theta_{\varphi, \chi^{+}}$with $G_{1}$ given here by

$$
G_{1}=\left.\left[c^{g}\left(D_{x_{n}}+i \partial_{x_{n}} \varphi^{g}\right), \mathrm{op}\left(\chi^{0}\right)\right] v^{g}\right|_{x_{n}=0^{+}}+\left.\left[c^{d}\left(D_{x_{n}}+i \partial_{x_{n}} \varphi^{d}\right), \mathrm{op}\left(\chi^{0}\right)\right] v^{d}\right|_{x_{n}=0^{+}}+\left.\mathrm{op}\left(\chi^{0}\right) \Theta_{\varphi}\right|_{x_{n}=0^{+}},
$$

and satisfying

$$
\left|G_{1}\right|_{0} \leq\left. C h|v|_{x_{n}=0^{+}}\right|_{0}+C\left|\Theta_{\varphi}\right|_{0} .
$$

We start by applying the method of Calderón projectors to the operator $P_{\varphi}^{g}$ and to $u^{g}$. We follow the same notation as in the proof of Proposition 2.9. We thus obtain an estimate of the form of (2.37), namely,

$$
\left\|u^{g}\right\|_{1} \leq C\left(\left\|P_{\varphi}^{g} v^{g}\right\|_{0}+h\left\|v^{g}\right\|_{1}+h^{\frac{1}{2}}\left(\left|\gamma_{1}\left(u^{g}\right)\right|_{0}+\left|\gamma_{0}\left(u^{g}\right)\right|_{1}\right)+\left.h^{2}\left|D_{x_{n}} v^{g}\right|_{x_{n}=0^{+}}\right|_{0}\right) .
$$

where $\gamma_{0}\left(u^{g}\right)=\left.u^{g}\right|_{x_{n}=0^{+}}$and $\gamma_{1}\left(u^{g}\right)=\left.D_{x_{n}} u^{g}\right|_{x_{n}=0^{+}}$. We also have the following trace equation, of the same form as (2.38),

$$
\gamma_{0}\left(u^{g}\right)=\mathrm{op}\left(a^{g}\right) \gamma_{0}\left(u^{g}\right)+\mathrm{op}\left(b^{g}\right) \gamma_{1}\left(u^{g}\right)+G_{2}^{g},
$$

with $a^{g} \in S_{\mathcal{T}}^{0}$ and $b^{g} \in S_{\mathcal{T}}^{-1}$, with principal symbols

$$
a_{0}^{g}=-\left.\left(\chi_{1} \frac{\rho^{g,-}}{\rho^{g,+}-\rho^{g,-}}\right)\right|_{x_{n}=0^{+}}, \quad b_{-1}^{g}=\left.\left(\chi_{1} \frac{1}{\rho^{g,+}-\rho^{g,-}}\right)\right|_{x_{n}=0^{+}},
$$

where $\chi_{1}\left(x_{0}, x, \xi_{0}, \xi^{\prime}\right) \in S_{\mathcal{T}}^{0}$, satisfies the same requirement as $\chi^{0}$, and is equal to one in a neighborhood of $\operatorname{supp}\left(\chi^{0}\right)$. The function $G_{2}^{g}$ satisfies

$$
\left|G_{2}^{g}\right|_{1} \leq C h^{-\frac{1}{2}}\left(\left\|P_{\varphi}^{g} v^{g}\right\|_{0}+h\left\|v^{g}\right\|_{1}+\left.h^{2}\left|D_{x_{n}} v^{g}\right|_{x_{n}=0^{+}}\right|_{0}\right) .
$$

We now use relation (2.68) in connection the transmission conditions $\left(\mathrm{TC}_{u}\right)$. With $\left(\mathrm{TC}_{u}\right)$, we write

$$
\mathrm{op}\left(b^{g}\right) \gamma_{1}\left(u^{g}\right)=-\mathrm{op}\left(b^{g}\right)\left(\beta \gamma_{1}\left(u^{d}\right)\right)+\mathrm{op}\left(b^{g}\right)\left(k \gamma_{0}\left(u^{d}\right)\right)+\mathrm{op}\left(b^{g}\right) \tilde{G}_{1}, \quad \gamma_{0}\left(u^{g}\right)=\gamma_{0}\left(u^{d}\right)+\theta_{\varphi, \chi^{0}},
$$

where $\beta=\left.\left(c^{d} / c^{g}\right)\right|_{x_{n}=0^{+}}, k=-i\left(\left.\partial_{x_{n}} \varphi^{g}\right|_{x_{n}=0^{+}}+\left.\beta \partial_{x_{n}} \varphi^{d}\right|_{x_{n}=0^{+}}\right)$and $\tilde{G}_{1}=-i \partial_{x_{n}} \varphi^{g} \theta_{\varphi, \chi^{0}}+\frac{1}{\left.c^{g}\right|_{x_{n}=0^{+}}} G_{1}$ that satisfies

$$
\left|\tilde{G}_{1}\right|_{0} \leq\left. C h|v|_{x_{n}=0^{+}}\right|_{0}+C\left(\left|\theta_{\varphi}\right|_{0}+\left|\Theta_{\varphi}\right|_{0}\right)
$$


by (2.66). From (2.68), we obtain

$$
(\underbrace{\mathrm{Id}-\mathrm{op}\left(a^{g}\right)-\mathrm{op}\left(b^{g}\right) \circ k}_{:=\mathrm{op}(k)}) \gamma_{0}\left(u^{d}\right)=-\mathrm{op}\left(b^{g}\right)\left(\beta \gamma_{1}\left(u^{d}\right)\right)+\mathrm{op}\left(b^{g}\right) \tilde{G}_{1}+\left(\mathrm{op}\left(a^{g}\right)-\mathrm{Id}\right) \theta_{\varphi, \chi^{0}}+G_{2}^{g},
$$

where $k$ stands here for the associated multiplication operator.

Let $\chi_{2}\left(x_{0}, x, \xi_{0}, \xi^{\prime}\right) \in S_{\mathcal{T}}^{0}$ satisfy the same requirement as $\chi^{0}$, and be equal to one in a neighborhood of $\operatorname{supp}\left(\chi^{0}\right)$ and be such that the symbol $\chi_{1}$ is equal to one in a neighborhood of $\operatorname{supp}\left(\chi_{2}\right)$. In $\operatorname{supp}\left(\chi_{2}\right)$, the principal symbol of $\kappa$ is given by

$$
\left.\kappa_{0}\right|_{\operatorname{supp}\left(\chi_{2}\right)}=\frac{\rho^{g,+}-k}{\rho^{g,+}-\rho^{g,-}} \in S_{\mathcal{T}}^{0} .
$$

In $\operatorname{supp}\left(\chi_{2}\right)$ we have $\operatorname{Im} \rho^{g,+} \geq C>0$ and $\operatorname{Im}(-k) \geq 0$ by Assumption 2.1, we see that $\kappa$ is elliptic in $\operatorname{supp}\left(\chi_{2}\right)$. Hence, there exists $l \in S_{\mathcal{T}}^{0}$, with $l=\sum_{j=0}^{M} h^{j} l_{j}$, with $l_{j} \in S_{\mathcal{T}}^{-j}$ and $l_{0}=\chi_{2} / \kappa_{0}$, such that

$$
\mathrm{op}\left(l_{M}\right) \circ \mathrm{op}(\kappa)=\mathrm{op}\left(\chi_{2}\right)+h^{M+1} R_{M}
$$

with $R_{M} \in \Psi_{\mathcal{T}}^{-1-M}$, for $M$ large. We thus obtain

$$
\gamma_{0}\left(u^{d}\right)=-\operatorname{op}(l) \circ \mathrm{op}\left(b^{g}\right)\left(\beta \gamma_{1}\left(u^{d}\right)\right)+G_{3},
$$

with

$$
G_{3}=\mathrm{op}(l) \circ \mathrm{op}\left(b^{g}\right) \tilde{G}_{1}+\mathrm{op}(l) \circ\left(\mathrm{op}\left(a^{g}\right)-\mathrm{Id}\right) \theta_{\varphi, \chi^{0}}+\mathrm{op}(l) G_{2}^{g}+\left(\mathrm{Id}-\mathrm{op}\left(\chi_{2}\right)\right) \gamma_{0}\left(u^{d}\right)-h^{M+1} R_{M} \gamma_{0}\left(u^{d}\right) .
$$

From the $\psi$ DO calculus, since $\operatorname{supp}\left(1-\chi_{2}\right) \cap \operatorname{supp}\left(\chi^{0}\right)=\emptyset$, we obtain

$$
\left|G_{3}\right|_{1} \leq C\left(h^{-\frac{1}{2}}\left\|P_{\varphi}^{g} v^{g}\right\|_{0}+h^{\frac{1}{2}}\|v\|_{1}+\left.h^{\frac{3}{2}}\left|D_{x_{n}} v^{g}\right|_{x_{n}=0^{+}}\right|_{0}+\left|\theta_{\varphi, \chi^{0}}\right|_{1}+\left|\Theta_{\varphi}\right|_{0}\right),
$$

by (2.70) and (2.69) and making use of the trace formula (2.32). We thus have

$$
\left|\gamma_{0}\left(u^{d}\right)\right|_{1} \leq C\left(\left|\gamma_{1}\left(u^{d}\right)\right|_{0}+h^{-\frac{1}{2}}\left\|P_{\varphi}^{g} v^{g}\right\|_{0}+h^{\frac{1}{2}}\|v\|_{1}+\left.h^{\frac{3}{2}}\left|D_{x_{n}} v^{g}\right|_{x_{n}=0^{+}}\right|_{0}+\left|\theta_{\varphi, \chi^{0}}\right|_{1}+\left|\Theta_{\varphi}\right|_{0}\right) .
$$

We now apply the Carleman method to the operator $P_{\varphi}^{d}$ and to $u^{d}$. By Assumption 2.1, and in particular by (2.3), and by Lemma 2 in [LR95] we have

$$
h\left\|u^{d}\right\|_{1}^{2}+\operatorname{Re}\left(h \mathscr{B}^{d}\left(u^{d}\right)+h^{2}\left(\left.\left(D_{n} u^{d}+L_{1}^{d} u^{d}\right)\right|_{x_{n}=0^{+}},\left.L_{0}^{d} u^{d}\right|_{x_{n}=0^{+}}\right)_{0}\right) \leq C\left\|P_{\varphi}^{d} u^{d}\right\|_{0}^{2},
$$

for $h$ sufficiently small, where $L_{1}^{d}, L_{0}^{d}$, and $\mathscr{R}^{d}$ are as given in the proof of Proposition 2.11. For any $\varepsilon>0$ we have

$$
h^{2}\left|\left(D_{n} u^{d}+L_{1}^{d} u^{d}, L_{0}^{d} u^{d}\right)_{0}\right| \leq h^{2}\left(\left|\gamma_{0}\left(u^{d}\right)\right|_{1}^{2}+\left|\gamma_{1}\left(u^{d}\right)\right|_{0}^{2}\right)
$$

With (2.71) we obtain

$$
\mathscr{B}^{d}\left(u^{d}\right)=\left(M^{*} \circ B^{d} \circ M \gamma_{1}\left(u^{d}\right), \gamma_{1}\left(u^{d}\right)\right)_{0}+U\left(\gamma_{1}\left(u^{d}\right), G_{3}\right),
$$

with

$$
M=\left(\begin{array}{c}
1 \\
-\Lambda^{1} \circ \mathrm{op}(l) \circ \mathrm{op}\left(b^{g}\right) \circ \beta
\end{array}\right) \in \Psi_{\mathcal{T}}^{0}
$$


with $\beta$ standing here for the associated multiplication operator, and where

$$
U\left(\gamma_{1}\left(u^{d}\right), G_{3}\right)=\left(B^{d} \circ M \gamma_{1}\left(u^{d}\right),\left(\begin{array}{c}
0 \\
\Lambda^{1} G_{3}
\end{array}\right)\right)_{0}+\left(B^{d}\left(\begin{array}{c}
0 \\
\Lambda^{1} G_{3}
\end{array}\right), M \gamma_{1}\left(u^{d}\right)\right)_{0}+\left(B^{d}\left(\begin{array}{c}
0 \\
\Lambda^{1} G_{3}
\end{array}\right),\left(\begin{array}{c}
0 \\
\Lambda^{1} G_{3}
\end{array}\right)\right) .
$$

With Young's inequality we obtain

$$
\begin{aligned}
\left|U\left(\gamma_{1}\left(u^{d}\right), G_{3}\right)\right| & \leq C\left(\left|\gamma_{1}\left(u^{d}\right)\right|_{0}\left|G_{3}\right|_{1}+\left|G_{3}\right|_{1}^{2}\right) \\
& \leq \varepsilon\left|\gamma_{1}\left(u^{d}\right)\right|_{0}^{2}+C_{\varepsilon}\left(h^{-1}\left\|P_{\varphi}^{g} v^{g}\right\|_{0}^{2}+h\|v\|_{1}^{2}+\left.h^{3}\left|D_{x_{n}} v^{g}\right|_{x_{n}=0^{+}}\right|_{0} ^{2}+\left|\theta_{\varphi, \chi^{0}}\right|_{1}^{2}+\left|\Theta_{\varphi}\right|_{0}^{2}\right),
\end{aligned}
$$

by (2.72).

In $\operatorname{supp}\left(\chi^{0}\right)$, the principal symbol of $M^{*} \circ B^{d} \circ M$ is in $S_{\mathcal{T}}^{0}$ and given by

$$
\begin{aligned}
\Sigma & =\sigma\left(M^{*} \circ B^{d} \circ M\right) \\
& =t^{t} \frac{\left.\left(\begin{array}{c}
1 \\
-\left\langle\left(\xi_{0}, \xi^{\prime}\right)\right\rangle^{1} l_{0} b_{-1}^{g} \beta
\end{array}\right)\left(\begin{array}{cc}
2 \partial_{x_{n}} \varphi^{d} & 2 q_{1}^{d}\left\langle\left(\xi_{0}, \xi^{\prime}\right)\right\rangle^{-1} \\
2 q_{1}^{d}\left\langle\left(\xi_{0}, \xi^{\prime}\right)\right\rangle^{-1} & -2 \partial_{x_{n}} \varphi^{d} q_{2}^{d}\left\langle\left(\xi_{0}, \xi^{\prime}\right)\right\rangle^{-2}
\end{array}\right)\left(\begin{array}{c}
1 \\
-\left\langle\left(\xi_{0}, \xi^{\prime}\right)\right\rangle^{1} l_{0} b_{-1}^{g} \beta
\end{array}\right)\right|_{x_{n}=0^{+}}}{}=\left.\left(2 \partial_{x_{n}} \varphi^{d}-4 q_{1}^{d} \beta \operatorname{Re}\left(l_{0} b_{-1}^{g}\right)-2 \beta^{2}\left|l_{0} b_{-1}^{g}\right|^{2} \partial_{x_{n}} \varphi^{d} q_{2}^{d}\right)\right|_{x_{n}=0^{+}} .
\end{aligned}
$$

In $\operatorname{supp}\left(\chi^{0}\right)$ we have

$$
\begin{aligned}
& \left.\left|l_{0} b_{-1}^{g}\right|^{-2}\right|_{x_{n}=0^{+}}=\left|\rho^{g,+}\right|_{x_{n}=0^{+}}-\left.k\right|^{2}=\left.\left(\left(\operatorname{Re} \rho^{g,+}\right)^{2}+\left(\operatorname{Im} \rho^{g,+}+\partial_{x_{n}} \varphi^{g}+\beta \partial_{x_{n}} \varphi^{d}\right)^{2}\right)\right|_{x_{n}=0^{+}}, \\
& \left.\operatorname{Re}\left(l_{0} b_{-1}^{g}\right)\left|l_{0} b_{-1}^{g}\right|^{-2}\right|_{x_{n}=0^{+}}=\left.\operatorname{Re} \rho^{g,+}\right|_{x_{n}=0^{+}} .
\end{aligned}
$$

We then obtain

$$
\begin{aligned}
\Sigma & =\left.2 \beta^{2}\left|l_{0} b_{-1}^{g}\right|^{2} \partial_{x_{n}} \varphi^{d}\left(\beta^{-2}\left|l_{0} b_{-1}^{g}\right|^{-2}-2 q_{1}^{d} \beta^{-1}\left(\partial_{x_{n}} \varphi^{d}\right)^{-1} \operatorname{Re} \rho^{g,+}-q_{2}^{d}\right)\right|_{x_{n}=0^{+}} \\
& =\left.2 \beta^{2}\left|l_{0} b_{-1}^{g}\right|^{2} \partial_{x_{n}} \varphi^{d}\left(\beta^{-2}\left(\left|l_{0} b_{-1}^{g}\right|^{-2}-\left(\operatorname{Re} \rho^{g,+}\right)^{2}\right)-\mu^{d}+\left(q_{1}^{d}\left(\partial_{x_{n}} \varphi^{d}\right)^{-1}-\beta^{-1} \operatorname{Re} \rho^{g,+}\right)^{2}\right)\right|_{x_{n}=0^{+}} \\
& \geq\left. 2 \beta^{2}\left|l_{0} b_{-1}^{g}\right|^{2} \partial_{x_{n}} \varphi^{d}\left(\beta^{-2}\left(\partial_{x_{n}} \varphi^{g}+\beta \partial_{x_{n}} \varphi^{d}\right)^{2}-\mu^{d}\right)\right|_{x_{n}=0^{+}} \geq C>0,
\end{aligned}
$$

by (2.63) and since

$$
\left.\left|l_{0} b_{-1}^{g}\right|^{2}\right|_{x_{n}=0^{+}} \geq C>0,\left.\quad\left|l_{0} b_{-1}^{g}\right|^{-2}\right|_{x_{n}=0^{+}}-\left.\left(\operatorname{Re} \rho^{g,+}\right)^{2}\right|_{x_{n}=0^{+}} \geq\left.\left(\partial_{x_{n}} \varphi^{g}+\beta \partial_{x_{n}} \varphi^{d}\right)^{2}\right|_{x_{n}=0^{+}},
$$

as $\left|\rho^{g,+}\right|$ remains bounded in $\operatorname{supp}\left(\chi^{0}\right)$ and by (2.77). Hence, Gårding's inequality yields [Tay81, Mar02]

$$
\operatorname{Re}\left(M^{*} \circ B^{d} \circ M \gamma_{1}\left(u^{d}\right), \gamma_{1}\left(u^{d}\right)\right)_{0} \geq C\left|\gamma_{1}\left(u^{d}\right)\right|_{0}^{2},
$$

for $h$ sufficiently small and $C>0$.

Combining (2.74), with (2.65), (2.73), (2.75), (2.76) and (2.78), for and $f$ and $\varepsilon$ sufficiently small we obtain

$$
h\left\|u^{d}\right\|_{1}^{2}+h\left|\gamma_{1}\left(u^{d}\right)\right|_{0}^{2}+h\left|\gamma_{0}\left(u^{d}\right)\right|_{1}^{2} \leq C\left(\left\|P_{\varphi} v\right\|_{0}^{2}+h^{2}\|v\|_{1}^{2}+\left.h^{4}\left|D_{x_{n}} v^{g}\right|_{x_{n}=0^{+}}\right|_{0} ^{2}+h\left|\theta_{\varphi}\right|_{1}^{2}+h\left|\Theta_{\varphi}\right|_{0}^{2}\right) .
$$

Note that the transmission conditions $\left(\mathrm{TC}_{u}\right)$ give

$$
\left|\gamma_{0}\left(u^{g}\right)\right|_{1}^{2}+\left|\gamma_{1}\left(u^{g}\right)\right|_{0}^{2} \leq C\left(\left|\gamma_{0}\left(u^{d}\right)\right|_{1}^{2}+\left|\gamma_{1}\left(u^{d}\right)\right|_{0}^{2}+\left|\theta_{\varphi}\right|_{1}^{2}+\left|\Theta_{\varphi}\right|_{0}^{2}+h\|v\|_{1}^{2}\right) \text {. }
$$


Recalling that

$$
D_{x_{n}} \mathrm{op}\left(\chi^{0} v\right)=\operatorname{op}\left(\chi^{0}\right) D_{x_{n}} v+\underbrace{\left[D_{x_{n}}, \mathrm{op}\left(\chi^{0}\right)\right]}_{\in h \Psi_{\mathcal{T}}^{0}} v
$$

we see that an appropriate linear combination of (2.67) and (2.79) then yields the sought partial Carleman estimate for $h$ sufficiently small.

2.6. Proof of Theorem 2.2. We choose $\chi^{+}, \chi^{-}$and $\chi^{0}$ with values in $[0,1]$ that satisfy the properties listed in Propositions 2.9, 2.11 and 2.13 respectively and furthermore $\chi^{+}+\chi^{-}+\chi^{0}=1$ in a neighborhood of $K \times \mathbf{R}^{n}$, which can be achieved by Proposition 2.7.

We recall that $v=e^{\Phi / h} w$. Since $1-\chi^{+}+\chi^{-}+\chi^{0}=0$ in a neighborhood of $\operatorname{supp}(w)$, we have

$$
\begin{gathered}
\left.\left|D_{x_{n}} v\right|_{x_{n}=0^{+}}\right|_{0} \leq\left.\left|\operatorname{op}\left(\chi^{+}\right) D_{x_{n}} v\right|_{x_{n}=0^{+}}\right|_{0}+\left.\left|\operatorname{op}\left(\chi^{-}\right) D_{x_{n}} v\right|_{x_{n}=0^{+}}\right|_{0}+\left.\left|\mathrm{op}\left(\chi^{0}\right) D_{x_{n}} v\right|_{x_{n}=0^{+}}\right|_{0}+\left.C h\left|D_{x_{n}} v\right|_{x_{n}=0^{+}}\right|_{0}, \\
\left.|v|_{x_{n}=0^{+}}\right|_{1} \leq\left.\left|\mathrm{op}\left(\chi^{+}\right) v\right|_{x_{n}=0^{+}}\right|_{1}+\left.\left|\mathrm{op}\left(\chi^{-}\right) v\right|_{x_{n}=0^{+}}\right|_{1}+\left.\left|\mathrm{op}\left(\chi^{0}\right) v\right|_{x_{n}=0^{+}}\right|_{1}+\left.C h|v|_{x_{n}=0^{+}}\right|_{1},
\end{gathered}
$$

and

$$
\|v\|_{1} \leq\left\|\mathrm{op}\left(\chi^{+}\right) v\right\|_{1}+\left\|\mathrm{op}\left(\chi^{-}\right) v\right\|_{1}+\left\|\mathrm{op}\left(\chi^{0}\right) v\right\|_{1}+C h\|v\|_{1} .
$$

These three inequalities together with (2.19), (2.46), and (2.64) then yield

$$
h\|v\|_{1}^{2}+\left.h|v|_{x_{n}=0^{+}}\right|_{1} ^{2}+\left.h\left|D_{x_{n}} v\right|_{x_{n}=0^{+}}\right|_{0} ^{2} \leq C\left(\left\|P_{\varphi} v\right\|_{0}^{2}+h\left|\theta_{\varphi}\right|_{1}^{2}+h\left|\Theta_{\varphi}\right|_{0}^{2}\right),
$$

for $h$ sufficiently small. Observing now that we have

$$
\left\|e^{\Phi / h} D_{x_{j}} w\right\|_{0} \leq\left\|D_{x_{j}}\left(e^{\Phi / h} w\right)\right\|_{0}+\left\|\partial_{x_{j}} \Phi e^{\Phi / h} w\right\|_{0},
$$

and similar inequalities for the norms at the interface $\left\{x_{n}=0^{+}\right\}$, and recalling the forms of $\theta_{\varphi}$ and $\Theta_{\varphi}$ in (2.11), we can conclude the proof of Theorem 2.2.

\section{Application to NUll controllability}

In this section, we prove an interpolation inequality from the Carleman estimate proven in the previous section. This will then yield the controllability result of Theorem 1.1.

With $\alpha \in\left(0, X_{0} / 2\right)$, we set $X=\left(0, X_{0}\right) \times \Omega, Y=\left(\alpha, X_{0}-\alpha\right) \times \Omega$.

Theorem 3.1. There exist $C \geq 0$ and $\delta \in(0,1)$ such that for $u \in H^{1}(X)$ that satisfies $(T C)$ and

$$
\begin{aligned}
& \left.u\right|_{\left(0, X_{0}\right) \times \Omega_{1}} \in H^{2}\left(\left(0, X_{0}\right), H^{2}\left(\Omega_{1}\right)\right) \quad \text { and }\left.\quad u\right|_{\left(0, X_{0}\right) \times \Omega_{2}} \in H^{2}\left(\left(0, X_{0}\right), H^{2}\left(\Omega_{2}\right)\right), \\
& \left.u\left(x_{0}, x\right)\right|_{x \in \partial \Omega}=0, x_{0} \in\left(0, X_{0}\right), \quad \text { and } \quad u(0, x)=0, x \in \Omega,
\end{aligned}
$$

we have

$$
\|u\|_{H^{1}(Y)} \leq C\|u\|_{H^{1}(X)}^{\delta}\left(\|A u\|_{L^{2}(X)}+\left\|\partial_{x_{0}} u(0, x)\right\|_{L^{2}(\omega)}\right)^{1-\delta} .
$$




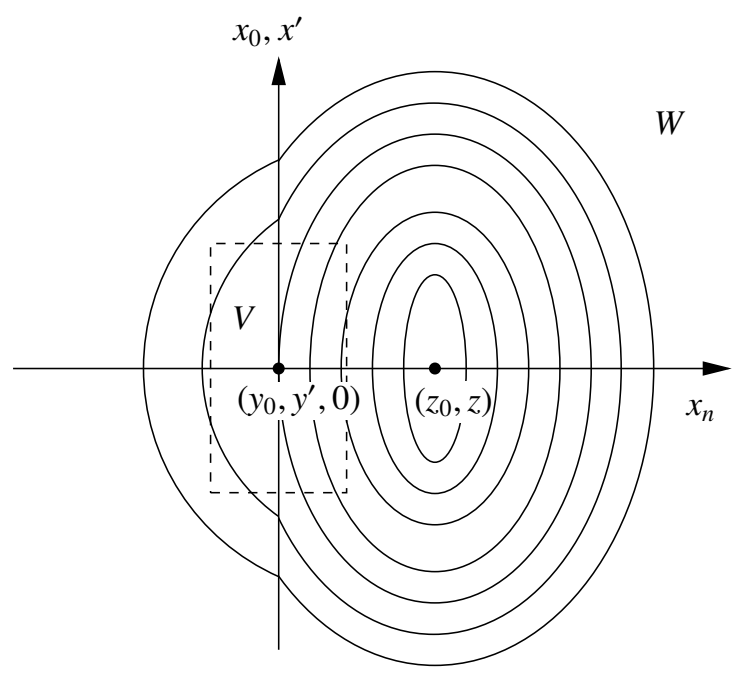

Figure 2: Level sets for the weight functions $\psi$ and $\varphi=e^{\lambda \psi}$ in local normal geodesic coordinates. The Carleman estimate of Theorem 2.2 can be applied in a region $V$ close to $\left(y_{0}, y^{\prime}, 0\right)$ (represented with a dashed line).

Before we sketch the proof of Theorem 3.1 we first indicate how the result of Theorem 1.1 follows.

Let $\phi_{j}, j \in \mathbf{N}$, be the eigenfunctions of the operator $L$ on $\Omega$, with Dirichlet boundary conditions, and let the associated eigenvalues be $\mu_{j}, j \in \mathbf{N}$, such that $0<\mu_{1} \leq \mu_{2} \leq \cdots \leq \mu_{k} \leq \ldots$. We let $\mu>0$ and we apply the interpolation inequality (3.1) to the function $w\left(x_{0}, x\right)=\sum_{\mu_{j} \leq \mu} a_{j} \frac{\sinh \left(\sqrt{\mu_{j}} x_{0}\right)}{\sqrt{\mu_{j}}} \phi_{j}(x)$, for $\left(a_{j}\right)_{j \in \mathbf{N}} \subset \mathbf{C}$. This yields Theorem 1.2, following for instance the proof given in [LZ98, Proof of Theorem 3]. This in turn yields Theorem 1.1 by the control construction method introduced in [LR95] (see also [LZ98, Section 5, Proposition 2] or [Mil06, Proof of Theorem 2.4 in Section 4.2]). As a consequence of Theorem 1.1, we have the following observability result.

Corollary 3.2. There exists a constant $C_{\mathrm{obs}} \geq 0$ such that the solution y to

$$
\begin{cases}-\partial_{t} y-L y=0 & \text { in } Q, \\ q=0 & \text { on }(0, T) \times \partial \Omega, \\ q(T)=q_{T} & \text { in } \Omega,\end{cases}
$$

with $q_{T} \in L^{2}(\Omega)$ satisfies $\|q(0)\|_{L^{2}(\Omega)}^{2} \leq C_{\mathrm{obs}}\|q\|_{L^{2}((0, T) \times \omega)}^{2}$.

Remark 3.3. With the technique used in [FI96], i.e., enlarging $\Omega$ in the neighborhood of part of $\partial \Omega$, we obtain a similar controllability (resp. observability) result for a localized boundary control (resp. observation).

3.1. Proof of the interpolation inequality. We first prove a local version of the interpolation inequality in a small neighborhood of a point $\left(y_{0}, y^{\prime}, 0\right)$ of the interface $\left(0, X_{0}\right) \times S$. 
We place ourselves in normal geodesic coordinates, as in Section 2, in a neighborhood $W$ of $\left(y_{0}, y, 0\right)$ and first construct a weight function $\psi$ in $W$. We start by defining the following anisotropic distance in $\mathbf{R}^{n+1}$ :

$$
\operatorname{dist}_{\alpha}\left(\left(a_{0}, a\right),\left(b_{0}, b^{\prime}\right)\right)=\left(\alpha\left\|\left(a_{0}, a^{\prime}\right)-\left(b_{0}, b^{\prime}\right)\right\|^{2}+\left|a_{n}-b_{n}\right|^{2}\right)^{\frac{1}{2}}, \quad \alpha>0 .
$$

Let $\left(z_{0}, z\right)=\left(y_{0}, y^{\prime}, z_{n}\right)$ be a point in $W$ away from the interface. We suppose $\left(z_{0}, z\right) \in W^{d}$, i.e., $z_{n}>0$ (for consistency with Section 2). For $\gamma>0$, we set

$$
\psi\left(x_{0}, x\right)= \begin{cases}-\operatorname{dist}_{\alpha}\left(\left(x_{0}, x\right),\left(z_{0}, z\right)\right) & \text { if } x_{n} \geq 0, \\ -\operatorname{dist}_{\alpha}\left(\left(x_{0}, x^{\prime}, \gamma x_{n}\right),\left(z_{0}, z\right)\right) & \text { if } x_{n}<0 .\end{cases}
$$

We note that $\psi$ is continuous across the interface $\left\{x_{n}=0\right\}$ and that

$$
\partial_{x_{n}} \psi\left(x_{0}, x\right)=\left(x_{n}-z_{n}\right)\left(\psi\left(x_{0}, x\right)\right)^{-1} \text { if } x_{n} \geq 0, \quad \text { and } \quad \partial_{x_{n}} \psi\left(x_{0}, x\right)=\gamma\left(\gamma x_{n}-z_{n}\right)\left(\psi\left(x_{0}, x\right)\right)^{-1} \text { if } x_{n} \leq 0,
$$

which yields $\left.\partial_{x_{n}} \psi\right|_{x_{n}=0^{-}}=\left.\gamma \partial_{x_{n}} \psi\right|_{x_{n}=0^{+}}$. We also have

$$
\partial_{x_{j}} \psi\left(x_{0}, x^{\prime}, 0\right)=\alpha\left(x_{j}-z_{j}\right)\left(\psi\left(x_{0}, x^{\prime}, 0\right)\right)^{-1}, \quad j=0, \ldots, n-1 .
$$

Note that $\left|x_{j}-z_{j}\right|$ is bounded in $W$ and that we can choose the parameter $\alpha$ sufficiently small to have $\left|\partial_{x_{j}} \psi\right|_{x_{n}=0} \mid, j=0, \ldots, n-1$ small as compared to $\left|\partial_{x_{n}} \psi\right|_{x_{n}=0^{+}} \mid$. We thus choose $\alpha$ and $\gamma$ sufficiently small to have $\psi$ satisfying the properties ${ }^{1}$ listed in Assumption 2.1 in a sufficiently small neighborhood $V$ of $\left(y_{0}, y\right)$, $V \subset W$, apart from the sub-ellipticity condition (2.3). Clearly $\left(z_{0}, z\right) \notin V$. Level sets for the function $\psi$ are represented in Figure 2.

We now note that the weight function $\varphi=e^{\lambda \psi}, \lambda>0$, also satisfies those conditions, possibly with different constants, from the homogeneity of the formulae in Assumption 2.1 w.r.t. to the weight function. The proof of Lemma 3 in [LR95, Section 3.B] then yields that $\varphi$ furthermore satisfies the sub-ellipticity condition (2.3) for $\lambda$ sufficiently large (see also Theorem 8.6.3 in [Hör63, Chapter 8] and Proposition 28.3.3 in [Hör85a, Chapter 28]). The local Carleman estimate of Theorem 2.2 then follows, with the weight function $\varphi$, for a possibly reduced neighborhood that we still denote by $V$ (see Proposition 2.7).

We choose $0<s_{1}<s_{1}^{\prime}$ and $0<\sigma<\sigma^{\prime}$ such that

$$
U^{\prime}=\left\{\left(x, x_{0}\right) ;\left\|\left(x_{0}, x^{\prime}\right)-\left(y_{0}, y^{\prime}\right)\right\|<s_{1}^{\prime},\left|x_{n}\right|<\sigma^{\prime}\right\} \subset V .
$$

We also set

$$
U=\left\{\left(x, x_{0}\right) ;\left\|\left(x_{0}, x^{\prime}\right)-\left(y_{0}, y^{\prime}\right)\right\|<s_{1},\left|x_{n}\right|<\sigma\right\} .
$$

We now choose $r_{1}<r_{1}^{\prime}<r_{2}<\psi\left(y_{0}, y\right)<r_{2}^{\prime}<r_{3}<r_{3}^{\prime}$, such that

$$
C_{1}=\left\{\left(x_{0}, x\right) \in \mathbf{R}^{n+1} ; \psi\left(x_{0}, x\right)=r_{1}\right\} \quad \text { and } \quad C_{3}^{\prime}=\left\{\left(x_{0}, x\right) \in \mathbf{R}^{n+1} ; \psi\left(x_{0}, x\right)=r_{3}^{\prime}\right\}
$$

satisfy $\mathcal{C}_{1} \cap \mathbf{R}_{-}^{n+1} \subset U, \mathcal{C}_{1} \cap \mathbf{R}_{+}^{n+1} \cap U \neq \emptyset$, which is equivalent to having

$$
-\left(\alpha s_{1}^{2}+z_{n}^{2}\right)^{\frac{1}{2}}<r_{1}
$$

and finally $C_{3}^{\prime} \cap U^{\prime} \subset\left\{x_{n} \leq \sigma\right\}$. We illustrate these choices in Figure 3 . We set $R_{j}=e^{\lambda r_{j}}, R_{j}^{\prime}=e^{\lambda r_{j}^{\prime}}$, $j=1,2,3$.

\footnotetext{
${ }^{1}$ depending on the case $(1,2$, or 3$)$ satisfied by the considered point $\left(y_{0}, y\right)$.
} 


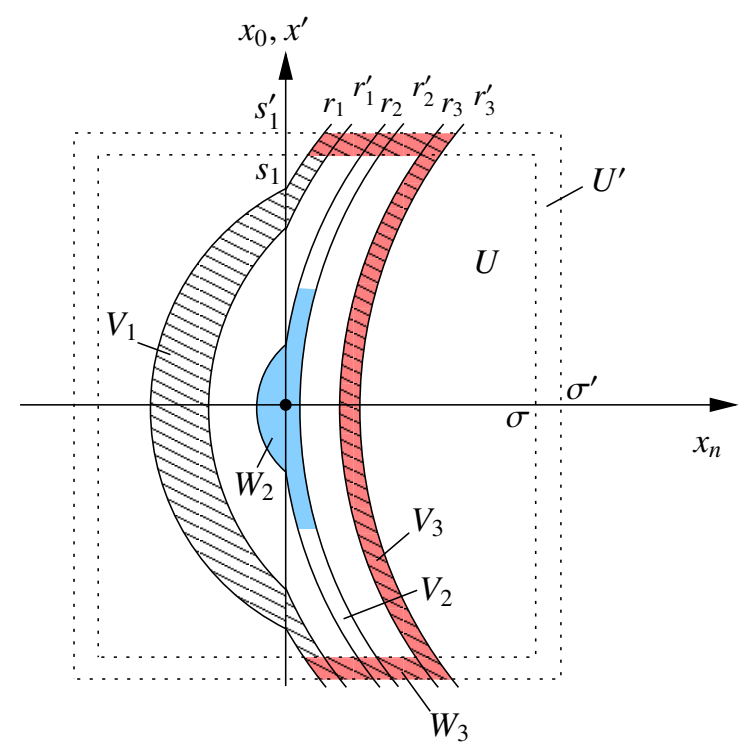

Figure 3: Neighborhoods around the point of interest for the proof of the interpolation inequality.

Following [LR95], we introduce

$$
V_{j}:=\left\{\left(x_{0}, x\right) \in U^{\prime} ; r_{j}<\psi\left(x_{0}, x\right)<r_{j}^{\prime}\right\}, \quad j=1,2,3
$$

and we further set

$$
\begin{aligned}
& V_{1^{\prime} \rightarrow 3}:=\left\{\left(x_{0}, x\right) \in U ; r_{1}^{\prime}<\psi\left(x_{0}, x\right)<r_{3}\right\}, \quad V_{1 \rightarrow 3^{\prime}}^{\prime}:=\left\{\left(x_{0}, x\right) \in U^{\prime} ; r_{1}<\psi\left(x_{0}, x\right)<r_{3}^{\prime}\right\} \\
& W_{3}=V_{3} \cup\left(V_{1 \rightarrow 3^{\prime}}^{\prime} \backslash U\right) .
\end{aligned}
$$

The region $W_{3}$ is represented shaded and stripped in Figure 3. With the choices we have made above, the region $W_{3}$ is contained in $\mathbf{R}_{+}^{n+1}$ and is finitely away from the interface $S=\left\{x_{n}=0\right\}$. We also choose $W_{2} \subset V_{2}$ such that $W_{2} \Subset U$. The region $W_{2}$ contains $\left(y_{0}, y^{\prime}, 0\right)$ and is represented shaded in Figure 3 .

We choose $\zeta \in \mathscr{C}_{c}^{\infty}\left(\mathbf{R}^{n+1}\right)$ such that $\zeta$ is equal to one on $V_{1^{\prime} \rightarrow 3}$ and vanishes outside $V_{1 \rightarrow 3^{\prime}}^{\prime}$. Then $\nabla_{x_{0}, x} \zeta$ vanishes outside $V_{1 \rightarrow 3^{\prime}}^{\prime} \backslash V_{1^{\prime} \rightarrow 3}$ which is the stripped region in Figure 3. For $u \in H^{1}(W)$ that satisfies (TC), we set $w=\zeta u$. Then $w$ satisfies the following transmission conditions

$$
\forall x_{0}, x^{\prime},\left.w\right|_{x_{n}=0^{-}}=\left.\left.w\right|_{x_{n}=0^{+}} \quad c \partial_{x_{n}} w\right|_{x_{n}=0^{-}}=\left.c \partial_{x_{n}} w\right|_{x_{n}=0^{+}}+\Theta
$$

where $\Theta=\left.\left(\left.c\right|_{x_{n}=0^{+}}-\left.c\right|_{x_{n}=0^{-}}\right)\left(u \partial_{x_{n}} \zeta\right)\right|_{x_{n}=0}$. Note that $\Theta$ is supported in $\left\{x_{n}=0\right\} \cap V_{1}$.

From the Carleman estimate of Theorem 2.2, after division by $h^{3}$, we have

$$
h^{-2}\left\|e^{\varphi / h} w\right\|_{0}^{2}+\left\|e^{\varphi / h} \nabla_{x_{0}, x} w\right\|_{0}^{2} \leq C\left(h^{1}\left\|e^{\varphi / h} A u\right\|_{0}^{2}+h^{1}\left\|e^{\varphi / h}[A, \zeta] u\right\|_{0}^{2}+\left|e^{\varphi / h} \Theta\right|_{0}^{2}\right), \quad 0<h \leq h_{0} .
$$

Note that $[A, \zeta]$ of order one and supported in $V_{1 \rightarrow 3^{\prime}}^{\prime} \backslash V_{1^{\prime} \rightarrow 3}$. We thus have

$$
\left\|e^{\varphi / h}[A, \zeta] u\right\|_{0} \leq C e^{R_{3}^{\prime} / h}\|u\|_{H^{1}\left(W_{3}\right)}+C e^{R_{1}^{\prime} / h}\|u\|_{H^{1}\left(V_{1}\right)} \leq C e^{R_{3}^{\prime} / h}\|u\|_{H^{1}\left(W_{3}\right)}+C e^{R_{1}^{\prime} / h}\|u\|_{H^{1}(W)} .
$$


We also have

$$
\left|e^{\varphi / h} \Theta\right|_{0} \leq C e^{R_{1}^{\prime} / h}\|u\|_{H^{1}(W)},
$$

by the trace formula. We thus obtain

$$
e^{R_{2} / h}\|u\|_{H^{1}\left(W_{2}\right)} \leq C e^{R_{1}^{\prime} / h}\|u\|_{H^{1}(W)}+C e^{R_{3}^{\prime} / h}\left(\|u\|_{H^{1}\left(W_{3}\right)}+\|A u\|_{L^{2}(W)}\right), \quad 0<h \leq h_{0} .
$$

Optimizing w.r.t. to $h$ as in [Rob95] we obtain $\mu_{0} \in(0,1)$ such that the local interpolation inequality

$$
\|u\|_{H^{1}\left(W_{2}\right)} \leq C\|u\|_{H^{1}(W)}^{1-\mu}\left(\|u\|_{H^{1}\left(W_{3}\right)}+\|A u\|_{L^{2}(W)}\right)^{\mu}
$$

holds for $0<\mu \leq \mu_{0}$. This inequality can be read as the "observation" of the $H^{1}$ norm of $u$ in the neighborhood of any point of the interface by the $H^{1}$ norm of $u$ in a neighborhood away from the interface and the $L^{2}$ norm of $A u$

Remark 3.4. As pointed above the region $W_{3}$ is contained in $\mathbf{R}_{+}^{n+1}$. The case $W_{3} \subset \mathbf{R}_{-}^{n+1}$ can naturally be obtained by changing $x_{n}$ into $-x_{n}$ in $W$.

Now that we have obtained such a local interpolation inequality at the interface, we can apply the procedure described in [LR95, pages 353-356] and prove the sought global interpolation inequality (3.1). See [LZ98, Proof of Theorem 3] to obtain the term $\left\|\partial_{x_{0}} u(0, x)\right\|_{L^{2}(\omega)}$ in the r.h.s. of (3.1). This concludes the proof of Theorem 3.1 .

Acknowledgements: We wish to thank G. Lebeau for encouraging discussions between the two authors on the subject of the article. We also thank Yves Dermenjian and Assia Benabdallah for many discussions on Carleman estimates for operators with non smooth coefficients and N. Lerner for discussions on Carleman estimates and unique continuation. This work was carried out when the first author was on research leaves at Laboratoire POems, INRIA Rocquencourt/ENSTA, CNRS UMR 2706, France and at Université Pierre et Marie Curie, Laboratoire Jacques-Louis Lions, CNRS UMR 7598, Paris, France. The first author was partially supported by l'Agence Nationale de la Recherche under grant ANR-07-JCJC-0139-01.

\section{REFERENCES}

[AE07] G. Alessandrini and L. Escauriaza, Null-controllability of one-dimensional parabolic equations, ESAIM Control Optim. Calc. Var., in press (2007).

[BDL07a] A. Benabdallah, Y. Dermenjian, and J. Le Rousseau, Carleman estimates for the one-dimensional heat equation with a discontinuous coefficient and applications to controllability and an inverse problem, J. Math. Anal. Appl. 336 (2007), $865-887$.

[BDL07b] _ On the controllability of linear parabolic equations with an arbitrary control location for stratified media, C. R. Acad. Sci. Paris, Ser I. 344 (2007), 357-362.

[Be103] M. Bellassoued, Carleman estimates and distribution of resonances for the transparent obstacle and application to the stabilization, Asymptotic Anal. 35 (2003), 257-279.

[DOP02] A. Doubova, A. Osses, and J.-P. Puel, Exact controllability to trajectories for semilinear heat equations with discontinuous diffusion coefficients, ESAIM: Control Optim. Calc. Var. 8 (2002), 621-661.

[FCZ02] E. Fernández-Cara and E. Zuazua, On the null controllability of the one-dimensional heat equation with BV coefficients, Comput. Appl. Math. 21 (2002), 167-190.

[FI96] A. Fursikov and O. Yu. Imanuvilov, Controllability of evolution equations, vol. 34, Seoul National University, Korea, 1996, Lecture notes. 
[Hör63] L. Hörmander, Linear Partial Differential Operators, Springer-Verlag, Berlin, 1963.

[Hör85a] _ _ The Analysis of Linear Partial Differential Operators, vol. IV, Springer-Verlag, 1985.

[Hör85b] _ _ The Analysis of Linear Partial Differential Operators, vol. III, Springer-Verlag, 1985, Second printing 1994.

[Hör90] _ The Analysis of Linear Partial Differential Operators, second ed., vol. I, Springer-Verlag, 1990.

[Le 07] J. Le Rousseau, Carleman estimates and controllability results for the one-dimensional heat equation with BV coefficients, J. Differential Equations 233 (2007), 417-447.

[Lio88] J.-L. Lions, Contrôlabilité Exacte, Perturbations et Stabilisation de Systèmes Distribués, vol. 1, Masson, Paris, 1988.

[LR95] G. Lebeau and L. Robbiano, Contrôle exact de l'équation de la chaleur, Comm. Partial Differential Equations 20 (1995), 335-356.

[LR97] _ Stabilisation de l'équation des ondes par le bord, Duke Math. J. 86 (1997), 465-491.

[LZ98] G. Lebeau and E. Zuazua, Null-controllability of a system of linear thermoelasticity, Arch. Rational Mech. Anal. 141 (1998), 297-329.

[Mar02] A. Martinez, An Introduction to Semiclassical and Microlocal Analysis, Springer-Verlag, 2002.

[Mil06] L. Miller, On the controllability of anomalous diffusions generated by the fractional laplacian, Mathematics of Control, Signals, and Systems 3 (2006), 260-271.

[Rob95] L. Robbiano, Fonction de coût et contrôle des solutions des équations hyperboliques, Asymptotic Anal. 10 (1995), 95-115.

[Tay81] M. E. Taylor, Pseudodifferential Operators, Princeton University Press, Princeton, New Jersey, 1981.

[Zui83] C. Zuily, Uniqueness and Non Uniqueness in the Cauchy Problem, Birkhauser, Progress in mathematics, 1983.

Universités d’ Aix-Marseille, Université de Provence, Laboratoire d’ Analyse Topologie Probabilités, CNRS UMR 6632, France. Current address: Université d'Orléans, Laboratoire Mathématiques et Applications, Physique Mathématique d’Orléans, CNRS UMR 6628, Fédération Denis Poisson, FR CNRS 2964, B.P. 6759, 45067 Orléans cedex 2, France.

Université de Versailles Saint-Quentin, Laboratoire de Mathématiques de Versailles, CNRS UMR 8100 , 45 Avenue des ÉtatsUnis, 78035 Versailles, France. 